\title{
ASYMPTOTICS OF ACCESSIBILITY SETS ALONG AN ABNORMAL TRAJECTORY
}

\author{
EMMANUEL TRÉLAT ${ }^{1}$
}

\begin{abstract}
We describe precisely, under generic conditions, the contact of the accessibility set at time $T$ with an abnormal direction, first for a single-input affine control system with constraint on the control, and then as an application for a sub-Riemannian system of rank 2. As a consequence we obtain in sub-Riemannian geometry a new splitting-up of the sphere near an abnormal minimizer $\gamma$ into two sectors, bordered by the first Pontryagin's cone along $\gamma$, called the $\mathrm{L}^{\infty}$-sector and the $\mathrm{L}^{2}$-sector. Moreover we find again necessary and sufficient conditions of optimality of an abnormal trajectory for such systems, for any optimization problem.
\end{abstract}

Mathematics Subject Classification. 93B03, 49K15.

Received November 8, 2000. Revised February 28, 2001.

\section{INTRODUCTION}

\subsection{Abnormal trajectories}

Consider a control system on $\mathbb{R}^{n}$ :

$$
\dot{x}(t)=f(x(t), u(t)), x(0)=x_{0}
$$

where $f: \mathbb{R}^{n} \times \mathbb{R}^{m} \longrightarrow \mathbb{R}^{n}$ is smooth, $x_{0} \in \mathbb{R}^{n}$, and the set of admissible controls $\mathcal{U}$ is made of measurable bounded functions $u:[0, T(u)] \longrightarrow \Omega \subset \mathbb{R}^{m}$.

Definition 1.1. Let $T>0$. The end-point mapping at time $T$ of system (1) is the mapping

$$
E_{T}: \begin{aligned}
\mathcal{U} & \longrightarrow \mathbb{R}^{n} \\
u & \longmapsto x_{u}(T)
\end{aligned}
$$

where $x_{u}$ is the trajectory associated to $u$.

It is a very classical fact that $E_{T}$ is smooth in the $\mathrm{L}^{\infty}$ topology, if $\mathcal{U} \subset \mathrm{L}^{\infty}([0, T])$.

Keywords and phrases: Accessibility set, abnormal trajectory, end-point mapping, single-input affine control system, subRiemannian geometry.

${ }^{1}$ Université de Bourgogne, Laboratoire de Topologie, UMR 5584 du CNRS, BP. 47870, 21078 Dijon Cedex, France; e-mail: trelat@topolog.u-bourgogne.fr 
Definition 1.2. The time-extended end-point mapping (see [6]) of system (1) is the mapping

$$
\begin{aligned}
\mathbb{R}^{+} \times \mathcal{U} & \longrightarrow \mathbb{R}^{n} \\
(T, u) & \longmapsto E_{T}(u)
\end{aligned}
$$

Definition 1.3. A control $u$ (or the corresponding trajectory $x_{u}$ ) is said to be abnormal on $[0, T]$ if $(T, u)$ is a singular point of the mapping $F$.

An equivalent definition may be given using the well-known Pontryagin Maximum Principle (see [21]), which parametrizes the previous condition:

Definition 1.4. A control $u$ on $[0, T]$ (or the corresponding trajectory $x_{u}$ ) is said to be abnormal if there exists a non trivial absolutely continuous function $p():.[0, T] \longrightarrow \mathbb{R}^{n} \times \mathbb{R}$ called adjoint vector, such that the trajectory $x$ satifies almost everywhere the system:

$$
\dot{x}=\frac{\partial H}{\partial p}, \dot{p}=-\frac{\partial H}{\partial x}, \frac{\partial H}{\partial u}=0
$$

where $H(x, p, u)=\langle p, f(x, u)\rangle$ is the Hamiltonian of the system, and moreover:

$$
H(x, p, u)=0 \quad \text { a.e. on }[0, T]
$$

Remark 1.5. If a control $u$ is abnormal on $[0, T]$ then it is abnormal on $[0, t]$ for any $t \in[0, T]$.

Definition 1.6. An abnormal control is said to be of corank 1 if the adjoint vector $p($.$) is defined uniquely up$ to a scalar multiplier.

Definition 1.7. Let $u$ be an abnormal control on $[0, T]$, and $x_{u}$ its associated trajectory. The subspace $\operatorname{im} d E_{t}(u)$ is called the first Pontryagin's cone at $x_{u}(t)$.

An abnormal control is of corank 1 on $[0, T]$ if the subspace im $d E_{T}(u)$ has codimension 1 in $\mathbb{R}^{n}$.

Remark 1.8. An abnormal control on $[0, T]$ is in particular a singular point of the end-point mapping $E_{T}$. Conversely if $u$ is a singularity of the end-point mapping and if moreover the trajectory $x_{u}$ associated to $u$ is almost everywhere tangent to its first Pontryagin's cone then $u$ is abnormal.

\subsection{Accessibility sets}

Definition 1.9. Consider the control system (1), and let $T>0$. The accessibility set at time $T$, denoted by $\operatorname{Acc}(T)$, is the set of points that can be reached from $x_{0}$ in time $T$ by solutions of system (1), i.e. this is the image of the end-point mapping $E_{T}$.

Let $\gamma$ be a reference trajectory on $[0, T]$, solution of (1), associated to a control $u$. Our aim is to describe $A c c(T)$ near $\gamma(T)$. If $u$ is not a singular point of the end-point mapping, then obviously $A c c(T)$ is open near $\gamma(T)$. The situation when $u$ is a critical point of $E_{T}$ has to be analyzed.

\subsection{Single-input affine systems}

Consider a smooth single-input affine system in $\mathbb{R}^{n}$ with constraint on the control:

$$
\begin{aligned}
& \dot{x}(t)=X(x(t))+u(t) Y(x(t)), \quad|u(t)| \leq \eta \\
& x(0)=x_{0}
\end{aligned}
$$


where $X, Y$ are smooth vector fields in $\mathbb{R}^{n}$, and $u$ is a scalar measurable function. Suppose that the trajectory $\gamma$ associated to the control $u=0$ is abnormal and of corank 1 on $[0, T]$. Let $p($.$) be an adjoint vector associated$ to $\gamma$. Then the condition $H=0$ is equivalent to:

$$
\langle p(t), X(\gamma(t))\rangle=0 \text { a.e. }
$$

i.e. $X \in \operatorname{im} d E_{t}(u)$ along $\gamma$.

The aim of this article is to describe precisely the accessibility set $A c c(T)$ at time $T$ near $\gamma(T)$.

The basic object we have to study is the so-called intrinsic second-order derivative of the end-point mapping:

Definition 1.10. The intrinsic second-order derivative along $\gamma$ is the real quadratic form:

$$
E_{u}^{\prime \prime}(v)=p(T) \cdot d^{2} E_{T}(u) \cdot(v, v)
$$

where $v \in \operatorname{ker} d E_{T}(u)$.

We shall make a spectral analysis of the end-point mapping along such an abnormal trajectory. Using the formalism and normal forms of [9] we shall represent the intrinsic second-order derivative by an explicit differential operator along the abnormal trajectory $\gamma$; then a spectral analysis of this operator shall lead to a precise description of the boundary of $A c c(T)$ near $\gamma(T)$ (Th. 2.10). In Section 2.2 we apply this result to sub-Riemannian systems of rank 2 (Th. 2.21), and obtain a new splitting-up of the sub-Riemannian sphere near an abnormal minimizer $\gamma$ into two sectors, in which the behaviours of minimizing trajectories near $\gamma$ are topologically different (Th. 2.26). On the other part this theory on accessibility sets leads to find again some well-known results on optimality of abnormal trajectories, that we recall and improve slightly in Section 3 .

\section{Asymptotics of the ACCESSibility Sets}

In this section we describe precisely the boundary of accessibility sets for a single-input affine system with constraint on the input near a reference abnormal trajectory. Then we apply our results to the sub-Riemannian case of rank 2 in order to get the contact of the sphere with the abnormal direction. As a consequence we obtain a splitting-up of the sphere into two sectors near the abnormal minimizer.

\subsection{Single-input affine control systems}

Consider a smooth single-input affine control system in $\mathbb{R}^{n}, n \geq 3$ :

$$
\dot{x}(t)=X(x(t))+u(t) Y(x(t)), x(0)=0
$$

with the constraint on the control

$$
|u(t)| \leq \eta
$$

Let $A c c^{\eta}(T)$ denote the accessibility set at time $T$ for this affine system with constraint $\eta$ on the control. Let $\gamma$ be a reference trajectory defined on $[0, T]$. In the sequel we make the following assumptions along $\gamma$ :

$\left(H_{0}\right) \gamma$ is injective, associated to $u=0$ on $[0, T]$.

$\left(H_{1}\right) \forall t \in[0, T] \quad K(t)=\operatorname{Vect}\left\{a d^{k} X . Y(\gamma(t)) / k \in \mathbb{N}\right\}$ (first Pontryagin's cone along $\gamma$ ) has codimension 1 , and is spanned by the first $n-1$ vectors, i.e.:

$$
K(t)=\operatorname{Vect}\left\{a d^{k} X . Y(\gamma(t)) / k=0 \ldots n-2\right\}
$$

$\left(H_{2}\right) \forall t \in[0, T] \quad a d^{2} Y . X(\gamma(t)) \notin K(t)$. 
$\left(H_{3}\right) \forall t \in[0, T] \quad X(\gamma(t)) \notin \operatorname{Vect}\left\{a d^{k} X . Y(\gamma(t)) / k=0 \ldots n-3\right\}$.

$\left(H_{4}\right) \forall t \in[0, T] \quad X(\gamma(t)) \in K(t)$.

In these conditions $\gamma$ is abnormal and of corank 1 . Actually assumptions $\left(H_{1}-H_{3}\right)$ are generic, see [10]. Moreover we get normal forms in which the intrinsic second-order derivative may be represented by an explicit differential operator (see [9]), in the following way.

Lemma 2.1. [9] Under the previous assumptions, the system $(X, Y)$ is in a $\mathrm{C}^{0}$-neighborhood of $\gamma$ feedbackequivalent to:

$$
\begin{aligned}
& f_{0}=\frac{\partial}{\partial x_{1}}+\sum_{i=1}^{n-2} x_{i+1} \frac{\partial}{\partial x_{i}}+\sum_{i, j=2}^{n} a_{i j}\left(x_{1}\right) x_{i} x_{j} \frac{\partial}{\partial x_{n}}+\sum_{i=1}^{n-1} x_{n} f_{i}\left(x_{1}\right) \frac{\partial}{\partial x_{i}}+\sum_{i=1}^{n} Z_{i} \frac{\partial}{\partial x_{i}} \\
& f_{1}=\frac{\partial}{\partial x_{n-1}}
\end{aligned}
$$

where $a_{n-1, n-1}(t)>0$ on $[0, T]$, and the 1-jet (resp. 2-jet) of $Z_{i}, i=1 \ldots n-1$ (resp. $Z_{n}$ ) along $\gamma$ is equal to 0 . Moreover the feedback $(\varphi, \alpha, \beta)$ satisfies:

$$
(\varphi, \alpha, \beta) \cdot(X, Y)=\left(f_{0}, f_{1}\right)
$$

where $f_{0}=\varphi_{*}(X+\alpha Y), f_{1}=\varphi_{*}(\beta Y)$, and $\varphi$ is a germ of diffeomorphism along $\gamma$ such that:

(i) $\varphi\left(x_{1}, 0, \ldots, 0\right)=\left(x_{1}, 0, \ldots, 0\right)$

(ii) $\frac{\partial \varphi}{\partial x_{n-1}}=(0, \ldots, 0, *, 0)$

and $\alpha, \beta$ are real functions defined in a neighborhood of $\gamma$ such that $\beta$ does not vanish along $\gamma$ and $\alpha_{\mid \gamma}=0$.

The $k$-jet is defined as follows:

Definition 2.2. Let $V=\sum_{k=1}^{n} V_{k} \frac{\partial}{\partial x_{k}}$ a vector field. Since $\gamma$ is given by $x_{1}(t)=t$ and $x_{i}(t)=0, i=2 \ldots n$, the component $V_{k}$ can be written in a neighborhood of $\gamma$ as $\sum_{p=1}^{+\infty} j_{p} V_{k}$, where $j_{0} V=V_{/ \gamma}$, and:

$$
j_{1} V_{k}=\sum_{i=2}^{n} a_{i}^{k}\left(x_{1}\right) x_{i}, j_{2} V_{k}=\sum_{i, j=2}^{n} b_{i j}^{k}\left(x_{1}\right) x_{i} x_{j}, \ldots
$$

Set $j_{i} V=\sum_{k=1}^{n} j_{i} V_{k} \frac{\partial}{\partial x_{k}}$. Then $\sum_{i=0}^{k} j_{i} V$ is called the $k$-jet of $V$ along $\gamma$.

Set $x_{1}=t+\xi$. In these conditions, the controllable part of the system is $\left(\xi, x_{2}, \ldots, x_{n-1}\right)$, the reference abnormal trajectory is $\gamma(t)=(t, 0, \ldots, 0)$, and the intrinsic second-order derivative $E_{0}^{\prime \prime}(v)$ along $\gamma$ is identified to:

$$
\begin{gathered}
E_{0}^{\prime \prime}(v)=\int_{0}^{T} \sum_{i, j=2}^{n-1} a_{i j}(t) \xi_{i}(t) \xi_{j}(t) \mathrm{d} t, \text { where: } \\
\dot{\xi}_{1}=\xi_{2}, \ldots, \dot{\xi}_{n-2}=\xi_{n-1}, \dot{\xi}_{n-1}=v, \quad \text { and } \quad \xi_{i}(0)=\xi_{i}(T)=0, i=1 \ldots, n-1 .
\end{gathered}
$$

Integrating by parts, it can be written into two different ways, namely either as a quadratic form in $\xi_{1}$ or as a quadratic form in $\xi_{2}$ : 
1. It is equal to $Q_{1 / G_{1}}\left(\xi_{1}\right)$, where:

$$
Q_{1}\left(\xi_{1}\right)=\int_{0}^{T} q_{1}\left(\xi_{1}\right) \mathrm{d} t \quad \text { and } \quad q_{1}\left(\xi_{1}\right)=\sum_{i, j=1}^{n-2} b_{i j} \xi_{1}^{(i)} \xi_{1}^{(j)}
$$

with $b_{i-1, j-1}=\frac{a_{i j}+a_{j i}}{2}$, and where $G_{1}$ is the following space corresponding to the kernel ker $d E_{T}(0)$ of the first derivative of the end-point mapping:

$$
G_{1}=\left\{\xi_{1} / \xi_{1}^{(2(n-2))} \in \mathrm{L}^{2}([0, T]), \xi_{1}^{(i)}(0)=\xi_{1}^{(i)}(T)=0, i=0 \ldots n-2\right\}
$$

Lemma 2.3. The quadratic form $Q_{1}$ is represented on $G_{1}$ by the operator $D_{1}$ so that:

$$
Q_{1}\left(\xi_{1}\right)=\left(\xi, D_{1} \xi\right)_{\mathrm{L}^{2}}
$$

where $\left(\right.$, ) is the usual scalar product in $\mathrm{L}^{2}([0, T])$, and:

$$
D_{1}=\frac{1}{2} \sum_{i=1}^{n-2}(-1)^{i} \frac{\mathrm{d}^{i}}{\mathrm{~d} t^{i}} \frac{\partial q_{1}}{\partial y^{(i)}}=\sum_{i, j=1}^{n-2}(-1)^{j} \frac{\mathrm{d}^{j}}{\mathrm{~d} t^{j}} b_{i j} \frac{\mathrm{d}^{i}}{\mathrm{~d} t^{i}}
$$

2. It is equal to $Q_{2 / G_{2}}\left(\xi_{2}\right)$, where:

$$
Q_{2}\left(\xi_{2}\right)=\int_{0}^{T} q_{2}\left(\xi_{2}\right) \mathrm{d} t \quad \text { and } \quad q_{2}\left(\xi_{2}\right)=\sum_{i, j=0}^{n-3} b_{i+1, j+1} x_{2}^{(i)} x_{2}^{(j)}
$$

and where $G_{2}$ is the space corresponding to the kernel of the first derivative:

$$
G_{2}=\left\{\xi_{2} / \xi_{2}^{(2(n-3))} \in \mathrm{L}^{2}([0, T]), \xi_{2}^{(i)}(0)=\xi_{2}^{(i)}(T)=0, i=0 \ldots n-3, \text { and } \int_{0}^{T} \xi_{2} \mathrm{~d} t=0\right\} .
$$

Lemma 2.4. The quadratic form $Q_{2}$ is represented on $G_{2}$ by the operator $D_{2}$ so that:

$$
Q_{2}\left(\xi_{2}\right)=\left(\xi_{2}, D_{2} \xi_{2}\right)_{\mathrm{L}^{2}}
$$

where

$$
D_{2}=\frac{1}{2} \sum_{i=0}^{n-3}(-1)^{i} \frac{\mathrm{d}^{i}}{\mathrm{~d} t^{i}} \frac{\partial q_{2}}{\partial y^{(i)}}=\sum_{i, j=0}^{n-3}(-1)^{j} \frac{\mathrm{d}^{j}}{\mathrm{~d} t^{j}} b_{i+1, j+1} \frac{\mathrm{d}^{i}}{\mathrm{~d} t^{i}}
$$

Note that $Q_{1}(\xi)=Q_{2}(\dot{\xi})$ and $D_{1}=-\frac{\mathrm{d}}{\mathrm{d} t} D_{2} \frac{\mathrm{d}}{\mathrm{d} t}$.

Our aim is to make a spectral analysis of these operators $D_{1}, D_{2}$. Unfortunately the spectrum of $D_{i}$ on $G_{i}$ is empty. Hence we shall enlarge the Sobolev space $G_{i}$ so that the spectrum is not trivial and that Representation Lemmas 2.3 and 2.4 are still valid. We set:

- $F_{1}=\left\{\xi_{1} / \xi_{1}^{(n-2)} \in \mathrm{L}^{2}([0, T]), \xi_{1}^{(i)}(0)=\xi_{1}^{(i)}(T)=0, i=0 \ldots n-3\right\}$ for the operator $D_{1}$. Endowed with the norm $\left\|\xi_{1}\right\|_{F_{1}}=\left\|\xi_{1}^{(n-2)}\right\|_{\mathrm{L}^{2}}, F_{1}$ is a Sobolev space.

- $F_{2}=\left\{\xi_{2} / \xi_{2}^{(n-3)} \in \mathrm{L}^{2}([0, T]), \xi_{2}^{(i)}(0)=\xi_{2}^{(i)}(T)=0, i=0 \ldots n-4\right\}$ for $D_{2}$ if $n \geq 4$ (if $n=3$, no condition is imposed). Endowed with the norm $\left\|\xi_{2}\right\|_{F_{2}}=\left\|\xi_{2}^{(n-3)}\right\|_{L^{2}}, F_{2}$ is a Sobolev space. 
Definition 2.5. Let $i=1$ or 2 . We call $T$ a conjugate time of $Q_{i}$ along $\gamma$ is there exists $\xi_{i} \in F_{i} \backslash\{0\}$ such that $\xi_{i}^{(2(n-1-i))} \in \mathrm{L}^{2}([0, T])$ and $D_{i} \xi_{i}=0$.

Lemma 2.6. (see for instance [13]) For any $f \in \mathrm{L}^{2}([0, T])$ ), if $T$ is not a conjugate time, there exists $\xi_{i} \in F_{i}$ unique such that $\xi_{i}^{(2(n-1-i))} \in \mathrm{L}^{2}([0, T])$ and $D_{i} \xi_{i}=f$. Let $L$ denote the operator $f \mapsto \xi_{i}$ considered as an operator from $\mathrm{L}^{2}([0, T])$ into $\mathrm{L}^{2}([0, T])$; it is selfadjoint and compact.

The following lemma is an improvement of [9], where only a non strict inequality is proved:

Lemma 2.7. Let $t_{c}$ (resp. $t_{c c}$ ) denote the first conjugate time of $Q_{1}$ on $F_{1}$ (resp. $Q_{2}$ on $F_{2}$ ). We have: $0<t_{c c}<t_{c}$.

Proof. This proof can be found in [9], where only a non strict inequality is proved. It is included only for convenience of the reader.

Let $y \in F_{1}$ such that $y^{(2(n-2))} \in \mathrm{L}^{2}([0, T])$, and $z=\dot{y}$; then $Q_{1}(y)=Q_{2}(z)$. Let $\lambda$ (resp. $\mu$ ) denote the smallest eigenvalue of $D_{1}$ (resp. $D_{2}$ ). From spectral properties of compact selfadjoint operators, we have:

$$
\lambda=\inf _{y \in F_{1}} \frac{Q_{1}(y)}{(y, y)} \quad \text { and } \quad \mu=\inf _{z \in F_{2}} \frac{Q_{2}(z)}{(z, z)} .
$$

By definition:

$$
\frac{Q_{1}(y)}{(y, y)}=\frac{Q_{2}(z)}{\int_{0}^{T}\left(\int_{0}^{t} z(s) \mathrm{d} s\right)^{2} \mathrm{~d} t}
$$

where $z \in F_{2}$ and $\int_{0}^{T} z(t) \mathrm{d} t=0$. Hence:

$$
\lambda \geq \inf \left\{\frac{Q_{2}(z)}{\int_{0}^{T}\left(\int_{0}^{t} z(s) \mathrm{d} s\right)^{2} \mathrm{~d} t} / z \in F_{2} \backslash\{0\}\right\} .
$$

Moreover we get from the Cauchy-Schwarz inequality:

$$
\int_{0}^{T}\left(\int_{0}^{t} z(s) \mathrm{d} s\right)^{2} \mathrm{~d} t \leq \int_{0}^{T} t \int_{0}^{t} z(s)^{2} \mathrm{~d} s
$$

and integrating by parts:

$$
\int_{0}^{T} t \int_{0}^{t} z(s)^{2} \mathrm{~d} s \mathrm{~d} t \leq \frac{T^{2}}{2} \int_{0}^{T} z(t)^{2} \mathrm{~d} t-\frac{1}{2} \int_{0}^{T} t^{2} z(t)^{2}<\frac{T^{2}}{2} \int_{0}^{T} z(t)^{2} \mathrm{~d} t
$$

Therefore:

$$
\lambda>\frac{2}{T^{2}} \inf \left\{\frac{Q_{2}(z)}{\int_{0}^{T} z(t)^{2} \mathrm{~d} t} / z \in F_{1}\right\}=\frac{2}{T^{2}} \mu .
$$

From [22] we know that eigenvalues of $D_{1}$ (or $D_{2}$ ) are continuous and decreasing functions of $T$. Hence if $T=t_{c}$ then $\lambda=0$, thus $\mu<0$ and consequently $t_{c c}<t_{c}$.

Remark 2.8. If $n=3$, we have $t_{c c}=+\infty$ provided assumptions $\left(H_{0}-H_{3}\right)$ are fulfilled on $\mathbb{R}^{+}$.

Remark 2.9. The notion of conjugate time does not depend on the constraint on the control. It comes from the fact that the abnormal reference control belongs to the interior of the domain of constraints.

The main result is the following. 
Theorem 2.10. Consider the affine system (5) with the constraint (6), and suppose that assumptions $\left(H_{0}-H_{4}\right)$ are fulfilled along the reference abnormal trajectory $\gamma$ on $[0, T]$. Let $t_{c c}$ and $t_{c}$ denote the first conjugate times associated to $\gamma$. Then:

1. There exist coordinates $\left(x_{1}, \ldots, x_{n}\right)$ locally along $\gamma$ such that in these coordinates: $\gamma(t)=(t, 0, \ldots, 0)$, and the first Pontryagin's cone along $\gamma$ is: $K(t)=\operatorname{Vect}\left\{\frac{\partial}{\partial x_{1}}, \ldots, \frac{\partial}{\partial x_{n-1}}\right\}_{\mid \gamma}$.

2. If $T$ is small enough then for any point $\left(x_{1}, \ldots, x_{n}\right)$ of $A c c^{\eta}(T) \backslash\{\gamma(T)\}$ close to $\gamma(T)$ we have: $x_{n}>0$ (see Fig. 1).

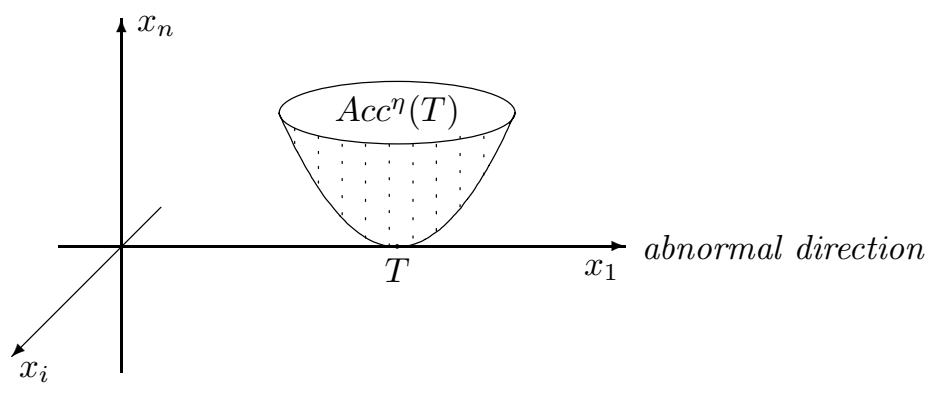

Figure 1. Shape of $A c c^{\eta}(T), T$ small.

3. If $T<t_{c}$, then in the plane $\left(x_{1}, x_{n}\right)$, near the point $(T, 0)$, the boundary of $A c c^{\eta}(T)$ does not depend on $\eta$, is a curve of class $\mathrm{C}^{2}$ tangent to the abnormal direction, and its first term is:

$$
x_{n}=A_{T}\left(x_{1}-T\right)^{2}+\mathrm{o}\left(\left(x_{1}-T\right)^{2}\right) .
$$

The function $T \mapsto A_{T}$ is continuous and strictly decreasing on $\left[0, t_{c}\left[\right.\right.$. It is positive on $\left[0, t_{c c}[\right.$ and negative on $] t_{c c}, t_{c}[$.

Moreover, if $\eta$ depends on $x_{1}-T$ then the result is still valid providing: $x_{1}-T=\mathrm{o}(\eta)$ as $x_{1} \rightarrow T$.

4. If $T>t_{c}$ then $\operatorname{Acc}^{\eta}(T)$ is open near $\gamma(T)$.

The evolution in function of $T$ of the intersection of $A c c^{\eta}(T)$ with the plane $\left(x_{1}, x_{n}\right)$ is represented in Figure 2. The contact with the abnormal direction is of order 2 ; the coefficient $A_{T}$ describes the concavity of the curve. Beyond $t_{c}$ the accessibility set is open.

Remark 2.11. The coefficient $A_{T}$ can be computed in the following way (see [9], and the proof just below). Actually there exists a function $J$ of class $\mathrm{C}^{2}(n-2)$ on $[0, T]$ such that $D_{1} J=0$ and satisfying the limit conditions:

$$
\forall k \in\{0, \ldots, n-3\} \quad J^{(k)}(0)=0, J^{(k)}(T)=\delta_{0}^{k} .
$$

Then:

$$
A_{T}=Q_{1}(J)
$$

Remark 2.12. Let us give a geometric interpretation of the role of the quadratic forms $Q_{1}, Q_{2}$.

1. Role of $Q_{1}$. On the one part note that $G_{1}$ is dense in $F_{1}$ and $Q_{1}$ is continuous on $F_{1}$ for the norm \|\|$_{F_{1}}$; hence the sign of $Q_{1}$ on $G_{1}$ is the same as on $F_{1}$. On the other part, from the definition of the first conjugate time $t_{c}$ and the extremal properties of selfadjoint compact operators, we get that $Q_{1 / F_{1}}$ is positive definite if $T<t_{c}$, and indefinite if $T>t_{c}$. Hence the same goes for $Q_{1 / G_{1}}$. 


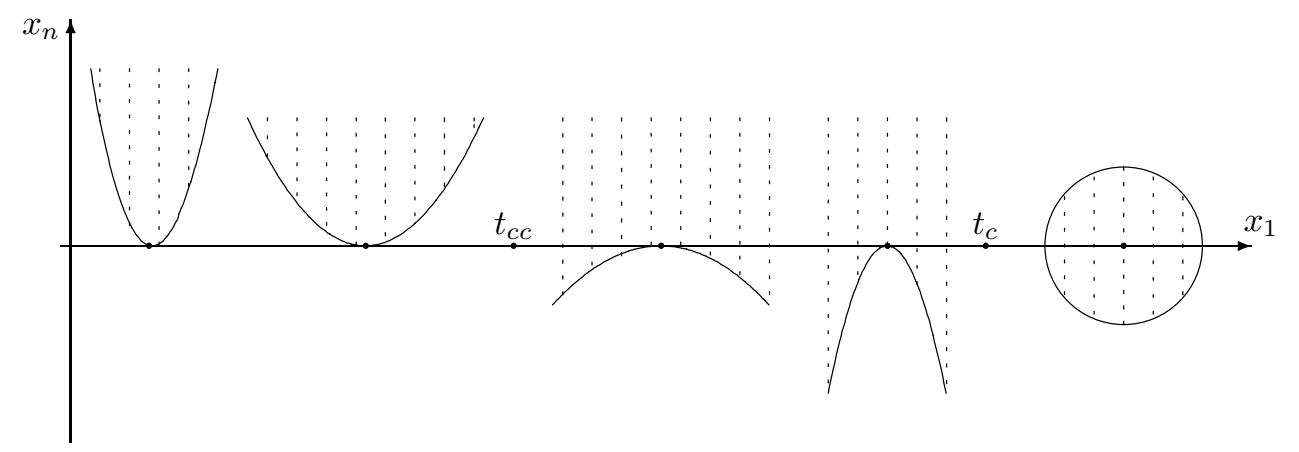

FIGURE 2

Now the interpretation of $Q_{1 / G_{1}}$ is the following: it is an equivalent of the coordinate $x_{n}(T)$ as all others coordinates are fixed: $x_{1}(T)=T, x_{2}(T)=\cdots=x_{n-1}(T)=0$.

As a consequence if $T<t_{c}$ then points $(T, 0, \ldots, 0, \varepsilon)$, where $\varepsilon \geq 0$ are attainable at time $T$. If $T>t_{c}$, then the same goes with $\varepsilon \geq 0$ or $\varepsilon \leq 0$.

2. Role of $Q_{2}$. Contrarily to the previous case, the subspace $G_{2}$ is a strict subspace of $F_{2}$. However, set:

$$
H_{2}=\left\{\xi_{2} / \xi_{2}{ }^{(2(n-3))} \in \mathrm{L}^{2}([0, T]), \xi_{2}{ }^{(i)}(0)=\xi_{2}{ }^{(i)}(T)=0, i=0 \ldots n-3\right\}
$$

Then $H_{2}$ is dense in $F_{2}$, and $Q_{2}$ is continuous on $F_{2}$ for the norm \|\|$_{F_{2}}$.

Hence using the same reasoning we obtain the following: if $T<t_{c c}$ then $Q_{2 / H_{2}}$ is positive definite; if $T>t_{c c}$ then $Q_{2 / H_{2}}$ is indefinite.

Now note that $Q_{2 / H_{2}}$ is an equivalent of the coordinate $x_{n}(T)$ as coordinates $x_{2}(T), \ldots, x_{n-1}(T)$ are fixed to 0 , but the coordinate $x_{1}(T)$ is let free.

As a consequence if $T<t_{c c}$ then points $(T \pm \delta, 0, \ldots, 0, \varepsilon)$, where $\varepsilon, \delta \geq 0$ are attainable at time $T$. If $T>t_{c c}$, then the same goes with $\varepsilon \geq 0$ or $\varepsilon \leq 0$.

This gives us the qualitative shape of $A c c(T)$. Actually this interpretation is enough to study the time-optimality of the abnormal trajectory $\gamma$, see Section 3.1.1. Here Theorem 2.10 gives more: it describes the $\operatorname{contact}$ of $A c c(T)$ with the abnormal direction.

Proof of Theorem 2.10. We shall recall briefly the formalism used by [9], which leads actually to a very precise decomposition of the intrinsic second-order derivative. It is based on normal forms of Lemma 2.1.

Let $D_{1}^{t}$ denote the operator (8) and $D_{2}^{t}$ the operator (9).

Lemma 2.13. For all $t \in[0, T]$ there exists an Hilbertian basis $\left(e_{n}^{t}\right)_{n \in \mathbb{N}}$ of $\mathrm{L}^{2}([0, T])$ such that

- $\forall n \in \mathbb{N} \quad e_{n}^{t}$ is $\mathrm{C}^{2}(n-2)$ and $e_{n}^{t} \in F_{1}$.

- $\forall n \in \mathbb{N} \quad D_{1}^{t} e_{n}^{t}=\lambda_{n}^{t} e_{n}^{t}$.

- $\lambda_{1}^{t} \leq \lambda_{2}^{t} \leq \cdots \leq \lambda_{n}^{t} \leq \cdots$

The eigenvalues $\lambda_{n}^{t}$ are continuous and decreasing functions of $t$, and $\lambda_{n}^{t} \underset{n \rightarrow+\infty}{\longrightarrow}+\infty$. Moreover, if $t_{c}$ denotes the first conjugate time of $D_{1}^{t}$, then:

- if $0 \leq t<t_{c}$ then $\lambda_{1}^{t}>0$,

- if $t>t_{c}$ then $\lambda_{1}^{t}<0$. 
Lemma 2.14. Let $0<t<t_{c}$. Then there exist $J_{i}^{t}, \bar{J}_{i}^{t}$ in $\mathrm{C}^{2}(n-2)([0, t]), i=1 \ldots n-2$, uniquely defined by the following equations:

(i) $D_{1}^{t} J_{i}^{t}=D_{1}^{t} \bar{J}_{i}^{t}=0, i=1 \ldots n-2$

(ii) $J_{i}^{(k)}(0)=\bar{J}_{i}^{(k)}(t)=0$.

$J_{i}^{(k)}(t)=\bar{J}_{i}^{(k)}(0)=\delta_{i-1}^{k}, k=0 \ldots n-3$.

Every $\xi$ in $\mathrm{C}^{2}(n-2)([0, t])$ can be expanded in a uniformly convergent series:

$$
\xi=\sum_{i=1}^{n-2} \alpha_{i} J_{i}^{t}+\sum_{i=1}^{n-2} \beta_{i} \bar{J}_{i}^{t}+\sum_{n=1}^{\infty} y_{n} e_{n}^{t}
$$

where $y_{n} \in \mathbb{R}, \alpha_{i}=\xi^{(i-1)}(0), \beta_{i}=\xi^{(i-1)}(t)$.

We shall use this lemma in the following way. In first approximation the system written in the normal form of Lemma 2.1 is given by:

$$
\dot{\xi}=x_{2}, \dot{x}_{2}=x_{3}, \ldots, \dot{x}_{n-1}=u
$$

except the last coordinate, which is given at order 2 by:

$$
\dot{x}_{n}=\sum_{i, j=2}^{n-1} a_{i j} x_{i} x_{j}
$$

Now the meaning of the $J_{i}, \bar{J}_{i}$ 's is clear: the functions $\bar{J}_{i}$ (resp. $J_{i}$ ) represent the initial (resp. final) conditions of the $n-2$ first coordinates. More precisely we have, see [9]:

Lemma 2.15. Let $T<t_{c}$, and $\xi=\sum_{i=1}^{\infty} v_{i} e_{i}^{T}+\sum_{i=1}^{n-2} \alpha_{i} J_{i}^{T}$. We have:

- $E_{i}^{T}\left(\xi^{(n-1)}\right)=\delta_{1}^{i} T+\alpha_{i}+\mathrm{o}\left(\left\|\xi^{(n-1)}\right\|_{\mathrm{L}^{\infty}}\right), i=1 \ldots n-2$.

- $\left.E_{n}^{T}\left(\xi^{(n-1)}\right)=Q_{1}^{T}(\xi)+\mathrm{o}\left(\| \xi^{(n-1)}\right) \|_{L^{\infty}}^{2}\right)$, where

$$
Q_{1}^{T}(\xi)=\sum_{i, j=1}^{n-2} A_{i j}^{T} \alpha_{i} \alpha_{j}+\sum_{i=1}^{\infty} \lambda_{i}^{T} v_{i}^{2}
$$

and $A_{i j}^{T}=\check{Q}_{1}^{T}\left(J_{i}^{T}, J_{j}^{T}\right)$, where $\check{Q}_{1}^{T}$ denotes the symmetric bilinear form associated to the quadratic form $Q_{1}^{T}$. In fact: $Q_{1}^{T}(\xi)=d^{2} E_{n}^{T}(0) .(u, u)$, where $u \sim \xi^{(n-1)}$, i.e.

$$
Q_{1}^{T}(\xi)=\int_{0}^{T} \sum_{i, j=1}^{n-2} b_{i j}(t) \xi^{(i)}(t) \xi^{(j)}(t) \mathrm{d} t
$$

where $b_{n-2, n-2}$ is continuous and strictly positive on $[0, T]$.

Hence with this formalism we study the image of the end-point mapping by constructing directly the trajectories (and not by choosing controls). Actually once a function $\xi$ has been determined, the corresponding control is $u=\xi^{(n-1)}+\mathrm{o}\left(\left\|\xi^{(n-1)}\right\|_{\mathrm{L}^{\infty}}\right)$.

First of all using expression (11) it is easy to prove point 2 of Theorem 2.10. Indeed to study the accessibility set at time $T$ from 0 we have to consider functions $\xi$ such that:

$$
\xi^{(i)}(0)=0, i=1 \ldots n-2 .
$$


Hence using repeatedly Poincaré's inequality ${ }^{2}$ we check that if $T$ is small enough then there exists $\beta>0$ such that:

$$
Q_{1}^{T}(\xi) \geq \beta \int_{0}^{T}\left(\xi^{(n-2)}(t)\right)^{2} \mathrm{~d} t
$$

We shall deduce the result by proving that actually $E_{n}^{T}(u)>0$ for the corresponding control $u$. The result would be immediate if $E_{n}^{T}$ were $\mathrm{C}^{2}$ in $H^{-1}$, i.e. in $\mathrm{L}^{2}$ topology for coordinate $x_{n-1}$. But this is wrong (for instance the function $f \mapsto \int_{0}^{T}\left(f^{2}+f^{3}\right) \mathrm{d} t$ is not $\mathrm{C}^{2}$ on $\left.\mathrm{L}^{2}\right)$. Anyway $E_{n}^{T}$ is a little better than $\mathrm{C}^{1}$. Precisely we have (see [4], Th. 7.1):

Lemma 2.16. $E_{n}^{T}(u)=Q_{1}^{T}(\xi)+R(\xi)$ where $|R(\xi)| \leq C\left\|\xi^{(n-2)}\right\|_{\mathrm{L}^{\infty}}\left\|\xi^{(n-2)}\right\|_{\mathrm{L}^{2}}^{2}$.

And hence if $T$ is small enough then for any point $\left(x_{1}, \ldots, x_{n}\right)$ belonging to $A c c^{\eta}(T) \backslash\{(T, 0, \ldots, 0)\}$ and to the $\mathrm{C}^{0}$-neighborhood of $\gamma$ of Lemma 2.1, we have: $x_{n}>0$.

So Lemma 2.15 describes the $n-2$ first coordinates and the last coordinate. Anyway [9] do not control the coordinate $E_{n-1}^{T}(u)$. Indeed their method consists in replacing the control $u \in \mathrm{L}^{2}$ by the control $x_{n-1} \in \mathrm{L}^{2}$, which is called Goh transformation and consists in fact in immersing $\mathrm{L}^{2}$ into the space $H^{-1}$, see [5]. Such trajectories are called generalized trajectories, and this corresponds to add to the set of controls $\mathrm{L}^{2}([0, T])$ Dirac measures at 0 and $T$.

Here, in order to take into consideration the constraint $|u| \leq \eta$ and to control the coordinate $E_{n-1}(u)$, the method used in [9] has to be adapted. The aim is to describe the boundary of the intersection of $A c c^{\eta}(T)$ with the plane $\left(x_{1}, x_{n}\right)$, close to the point $(T, 0)$ (corresponding to $\gamma(T)$ ). Let $x$ be a real close to $T$. We have to solve equations:

$$
E_{1}^{T}(u)=x, E_{2}^{T}(u)=\cdots=E_{n-1}^{T}(u)=0
$$

in the domain $|u| \leq \eta$, and then among such solutions we have to minimize the last coordinate $E_{n}^{T}(u$ ) (in order to describe the boundary of the accessibility set). We proceed in the following way. Set $x_{1}(t)=t+\xi(t)$; we shall first determine $\xi$ such that the control $u=\xi^{(n-1)}$ satisfies (12). From Lemma 2.14, $\xi$ can be expanded in:

$$
\xi=\sum_{i=1}^{n-2} \alpha_{i} J_{i}^{T}+\sum_{i=1}^{\infty} v_{i} e_{i}^{T}
$$

From Lemma $(2.15)$, the first $n-2$ coordinates are, if $u \sim \xi^{(n-1)}=\sum_{i=1}^{n-2} \alpha_{i} J_{i}^{T(n-1)}+v$ :

$$
\begin{aligned}
E_{1}^{T}(u) & =T+\alpha_{1}+\mathrm{o}\left(\alpha_{1}, \ldots, \alpha_{n-2},\|v\|_{\mathrm{L}^{\infty}}\right) \\
E_{2}^{T}(u) & =\alpha_{2}+\mathrm{o}\left(\alpha_{1}, \ldots, \alpha_{n-2},\|v\|_{\mathrm{L}^{\infty}}\right) \\
& \vdots \\
E_{n-2}^{T}(u) & =\alpha_{n-2}+\mathrm{o}\left(\alpha_{1}, \ldots, \alpha_{n-2},\|v\|_{\mathrm{L}^{\infty}}\right) .
\end{aligned}
$$

Let us solve equations (12). We get from the Implicit Function theorem:

$$
\begin{aligned}
\alpha_{1} & =x-T+\mathrm{o}\left(x-T,\|v\|_{\mathrm{L}^{\infty}}\right) \\
\alpha_{2} & =\mathrm{o}\left(x-T,\|v\|_{\mathrm{L}^{\infty}}\right) \\
& \vdots \\
\alpha_{n-2} & =\mathrm{o}\left(x-T,\|v\|_{\mathrm{L}^{\infty}}\right)
\end{aligned}
$$

${ }^{2} f(0)=0 \Longrightarrow\|f\|_{L^{\infty}([0, T])} \leq \sqrt{T}\left\|f^{\prime}\right\|_{L^{2}([0, T])}$. 
as $x \rightarrow T$ and $v \stackrel{\mathrm{L}^{\infty}}{\longrightarrow} 0$. Further we will modify $u$ in order to get also:

$$
x_{n-1}(0)=x_{n-1}(T)=0 \quad \text { and } \quad|u| \leq \eta \text {. }
$$

Let us first study the last coordinate. We get from Lemma 2.15:

$$
E_{n}^{T}(u)=A_{T}(x-T)^{2}+\sum_{i=1}^{\infty} \lambda_{i}^{T} v_{i}^{2}+\mathrm{o}\left(\left(x-T+\|v\|_{\mathrm{L}^{\infty}}\right)^{2}\right)
$$

as $x \rightarrow T$ and $v \stackrel{L^{\infty}}{\longrightarrow} 0$. In this expression we can see that the minimum is reached at $v=0$ up to $a \mathrm{o}\left((x-T)^{2}\right)$, for if $T<t_{c}$ then all $\lambda_{i}^{T}$ 's are positive (Lem. 2.13).

Remark 2.17. If we do not neglect any term then the minimum is not necessarily reached at $v=0$ because of terms of order 3 in $(x-T)^{2} v_{i}$.

Therefore, at order 2 , the minimum is reached at $u \sim \sum_{i=1}^{n-1} \alpha_{i} J_{i}^{T^{(n-1)}}$ and equals:

$$
E_{n}^{T}(u)=A_{T}(x-T)^{2}+\mathrm{o}\left((x-T)^{2}\right) \quad \text { as } x \rightarrow T .
$$

We shall now prove that it is possible to modify $u$, taking into account the constraint $|u| \leq \eta$, in order to obtain $x_{n-1}(0)=x_{n-1}(T)=0$, without changing the previous results on the other coordinates.

First of all, if $x$ is close enough to $T$, then the $\alpha_{i}$ 's are small and thus the constraint $|u| \leq \eta$ is satisfied.

Let us modify $u$ (and hence $x_{n-1}$ ) in the following way. Set $u(t)=\eta$ if $x_{n-1}(0)>0$ or $-\eta$ if $x_{n-1}(0)<0$ on $\left[0, t_{1}\right]$ (same construction on $\left[t_{2}, T\right]$ ), where $t_{1}$ and $t_{2}$ are the coinciding times, i.e. the times at which $x_{n-1}$ coincides with its initial graph, see Figure 3.

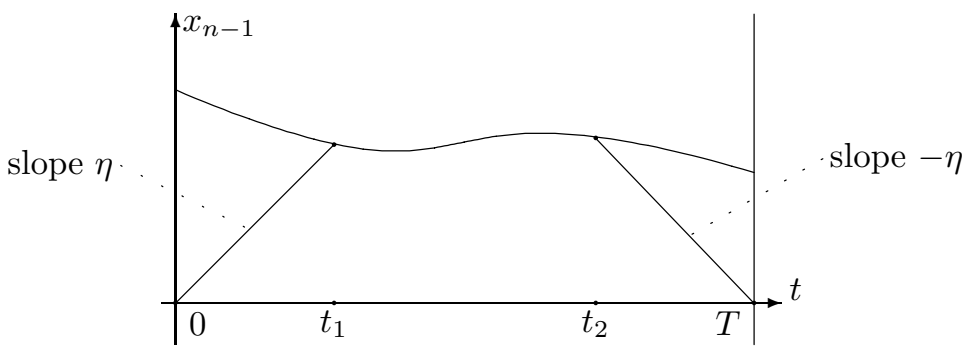

Figure 3

Precisely:

- if $0 \leq t \leq t_{1}:|u(t)|=\eta,\left|x_{n-1}(t)\right|=\eta t$, and the coinciding time $t_{1}$ is such that $\eta t_{1} \sim\left|\sum_{i=1}^{n-2} \alpha_{i} J_{i}^{T^{(n-2)}}\left(t_{1}\right)\right|$;

- if $t_{1} \leq t \leq t_{2}: u(t) \sim \xi^{(n-1)}(t), x_{n-1}(t)=\sum_{i=1}^{n-2} \alpha_{i} J_{i}^{T^{(n-2)}}(t)$

- if $t_{2} \leq t \leq T:|u(t)|=\eta,\left|x_{n-1}(t)\right|=\eta(T-t)$, where $t_{2}$ is such that $\eta\left(T-t_{2}\right) \sim\left|\sum_{i=1}^{n-2} \alpha_{i} J_{i}^{T^{(n-2)}}\left(t_{2}\right)\right|$. 
We shall now check that if

$$
x-T=\mathrm{o}(\eta) \quad \text { as } x \rightarrow T
$$

then all previous results are still valid.

Clearly: $\eta t_{1}=\mathrm{O}(x-T)$ and $\eta\left(t_{2}-T\right)=\mathrm{O}(x-T)$ as $x \rightarrow T$. Moreover, at order 1, the system is:

$$
\dot{x}_{1}=1+x_{2}, \dot{x}_{2}=x_{3}, \ldots, \dot{x}_{n-1}=u .
$$

Therefore:

$$
x\left(t_{1}\right)-t_{1} \sim \eta \frac{t_{1}^{n-1}}{(n-1) !} \quad \text { and } \quad x_{1}\left(t_{2}\right)-t_{2}-x_{1}(T)+T \sim \eta \frac{t_{2}^{n-1}}{(n-1) !} .
$$

Now if $n \geq 3$ and $x-T=\mathrm{o}(\eta)$ then these terms are negligibly small in comparison to $x-T$.

As concerns the last coordinate, we obtain:

$$
x_{n}\left(t_{1}\right)=\mathrm{O}\left(\eta^{2} t_{1}^{3}\right)=\mathrm{o}\left((x-T)^{2}\right) \quad \text { and } \quad x_{n}(T)-x_{n}\left(t_{2}\right)=\mathrm{O}\left(\eta^{2} t_{2}^{3}\right)=\mathrm{o}\left((x-T)^{2}\right) .
$$

In these conditions, all our previous construction is still valid. Hence in the plane $\left(x_{1}, x_{n}\right)$ the boundary of $\operatorname{Acc}^{\eta}(T)$ is a curve of class $C^{2}$, independant of the constraint, such that $x_{n} \sim A_{T}\left(x_{1}-T\right)^{2}$, which proves Theorem 2.10. Moreover it results from [9] and [22] that the function $T \mapsto A_{T}$ is continuous and decreasing on $\left[0, t_{c}\left[\right.\right.$, positive on $\left[0, t_{c c}[\right.$ and negative on $] t_{c c}, t_{c}[$.

\subsection{Application to the sub-Riemannian case}

\subsubsection{Asymptotics of the sub-Riemannian sphere along an abnormal direction}

Consider a smooth sub-Riemannian structure $(M, \Delta, g)$ where $M$ is a Riemannian $n$-dimensional manifold, $n \geq 3, \Delta$ is a rank 2 distribution on $M$, and $g$ is a metric on $\Delta$. Let $x_{0} \in M$; our point of view is local and we can assume that $M=\mathbb{R}^{n}$ and $x_{0}=0$. Suppose there exists a smooth injective abnormal trajectory $\gamma$ passing through 0 . Up to changing coordinates and reparametrizing we can assume that:

- $\gamma(t)=(t, 0, \ldots, 0)$;

- $\Delta=\operatorname{Span}\{X, Y\}$ where $X, Y$ are $g$-orthonormal;

- $\gamma$ is the integral curve of $X$ passing through 0.

Under these assumptions, the sub-Riemannian problem is equivalent to the time-optimal problem for the system:

$$
\dot{x}=v X(x)+u Y(x), x(0)=0
$$

where the controls $v, u$ satisfy the constraint:

$$
v^{2}+u^{2} \leq 1
$$

The reference abnormal trajectory $\gamma$ corresponds to the control: $v=1, u=0$.

Let us now define a notion of constrained accessibility set:

Definition 2.18. Let $0<\alpha<1$. We denote by $\operatorname{Acc}_{S R}^{\alpha}(T)$ the accessibility set at time $T$ for the sub-Riemannian system (14) with the additional constraint on the control:

$$
v^{2}+u^{2} \leq 1,1-\alpha \leq v \leq 1,|u| \leq \alpha
$$

(see Fig. 4).

Note that controls steering 0 to points of $A c c_{S R}^{\alpha}(T)$ are in a $\alpha$-neighborhood in $\mathrm{L}^{\infty}$ metric of the abnormal reference control $v=1, u=0$. 


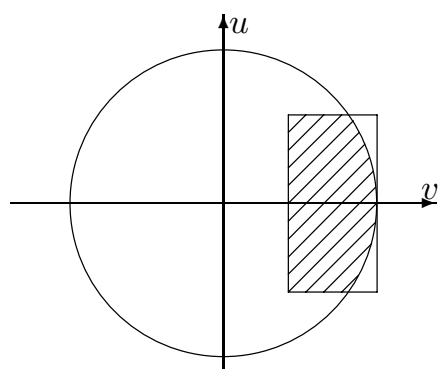

Figure 4

Definition 2.19. We call affine system associated to the sub-Riemannian system (14) the following system:

$$
\dot{x}=X(x)+w Y(w)
$$

where the control $w$ satisfies a constraint of the form: $|w| \leq \eta$.

Let $A c c_{A}^{\eta}(T)$ denote the accessibility set at time $T$ for this affine system with the constraint: $|w| \leq \eta$. The reference trajectory $\gamma$ corresponds to $w=0$, and is also abnormal for this affine system.

The following lemma gives a precise comparison of constrained accessibility sets of systems (14) and (16):

Lemma 2.20. $\quad$ 1. $\forall \alpha \in] 0,1\left[\quad \operatorname{Acc}_{S R}^{\alpha}(T) \subset \bigcup_{(1-\alpha) T \leq s \leq T} \operatorname{Acc}_{A}^{\frac{\alpha}{1-\alpha}}(s)\right.$.

$$
\text { 2. } \bigcup_{T_{0} \leq s \leq \frac{T}{\sqrt{1+\eta^{2}}}} A c c_{A}^{\eta}(s) \subset A c c_{S R}^{\alpha}(T) \quad \text { where } \alpha=\max \left(1-\frac{T_{0}}{T}, \frac{\eta}{\sqrt{1+\eta^{2}}}\right) .
$$

Proof. Let us prove the first inclusion. If $x_{1} \in A c c_{S R}^{\alpha}(T)$ then there exists a control $(v, u)$ such that $v^{2}+u^{2} \leq 1$, $1-\alpha \leq v \leq 1,|u| \leq \alpha$, and such that the corresponding trajectory satisfies:

$$
\dot{x}=v X+u Y, x(0)=0, x(T)=x_{1} .
$$

As $\alpha<1, v$ does not vanish, the following reparametrizing holds: $\frac{\mathrm{d} s}{\mathrm{~d} t}=v$. Set $y(s)=x(t), w(s)=\frac{u(t)}{v(t)}$, and $S=\int_{0}^{T} v$. Then: $x_{1}=x(T)=y(S)$, and $S$ is such that:

$$
(1-\alpha) T \leq S \leq T
$$

Moreover:

where $|w| \leq \frac{\alpha}{1-\alpha}$. Therefore:

$$
\frac{\mathrm{d} y}{\mathrm{~d} s}=X+\frac{u}{v} Y=X+w Y
$$

which proves the first part of the lemma.

$$
x_{1} \in \bigcup_{(1-\alpha) T \leq s \leq T} A c c_{A}^{\frac{\alpha}{1-\alpha}}(s)
$$

Let us now check the second inclusion. Let $S \in\left[T_{0}, \frac{T}{\sqrt{1+\eta^{2}}}\right]$ and $x_{1} \in \operatorname{Acc}_{A}^{\eta}(S)$. There exists a control $w$ such that $|w| \leq \eta$ and the corresponding trajectory satisfies:

$$
\frac{\mathrm{d} y}{\mathrm{~d} s}=X+w Y, y(0)=0, y(S)=x_{1}
$$


Let $\varepsilon>0$ such that $T=\frac{S}{1-\varepsilon}$. Let us make the reparametrizing: $\frac{\mathrm{d} s}{\mathrm{~d} t}=1-\varepsilon$, and set: $x(t)=y(s), v(t)=1-\varepsilon$, $u(t)=(1-\varepsilon) w(s)$, where $t \in[0, T]$. Then:

$$
\dot{x}=v X+u Y, x(0)=0, x(T)=y(S)=x_{1} .
$$

Let us now check the constraint on the control $(v, u)$. By definition: $T_{0} \leq S \leq \frac{T}{\sqrt{1+\eta^{2}}}$ and $S=(1-\varepsilon) T$. Hence:

$$
1-\varepsilon \leq \frac{1}{\sqrt{1+\eta^{2}}} \quad \text { and } \quad 1-\varepsilon \geq \frac{T_{0}}{T}
$$

and thus:

Moreover:

$$
1-\left(1-\frac{T_{0}}{T}\right) \leq v \leq 1 \quad \text { and } \quad|u| \leq \frac{\eta}{\sqrt{1+\eta^{2}}}
$$

Therefore we can conclude that:

$$
v^{2}+u^{2} \leq(1-\varepsilon)^{2}\left(1+\eta^{2}\right) \leq 1
$$

$$
x_{1} \in \operatorname{Acc}_{S R}^{\alpha}(T) \quad \text { where } \quad \alpha=\max \left(1-\frac{T_{0}}{T}, \frac{\eta}{\sqrt{1+\eta^{2}}}\right) .
$$

Using the previous lemma and Theorem 2.10 we can prove the following:

Theorem 2.21. Suppose assumptions $\left(H_{0}-H_{3}\right)$ are fulfilled along the reference abnormal trajectory $\gamma$ for the system $(X, Y)$. Let $t_{c c}$ and $t_{c}$ denote the first conjugate times of $\gamma$ for the associated affine system. Let $\left.\alpha \in\right] 0,1[$. Then:

1. There exist coordinates $\left(x_{1}, \ldots, x_{n}\right)$ locally along $\gamma$ such that in these coordinates: $\gamma(t)=(t, 0, \ldots, 0)$, and the first Pontryagin's cone along $\gamma$ is: $K(t)=\operatorname{Vect}\left\{\frac{\partial}{\partial x_{1}}, \ldots, \frac{\partial}{\partial x_{n-1}}\right\}_{\left.\right|_{\gamma}}$.

2. If $T$ is small enough then for any point $\left(x_{1}, \ldots, x_{n}\right)$ of $\operatorname{Acc}_{S R}^{\alpha}(T)$ close to $\gamma(T)$ we have $x_{n} \geq 0$ (see Fig. 1).

3. If $T<t_{c c}$, then in the plane $\left(x_{1}, x_{n}\right)$, close to the point $(T, 0)$, the boundary of $A c c_{S R}^{\alpha}(T)$ does not depend on $\alpha$, is a curve of class $\mathrm{C}^{2}$ outside $(T, 0)$, tangent to the abnormal direction, whose first term is:

- if $x_{1} \leq T$ then $x_{n}=0$;

- if $x_{1} \geq T$ then $x_{n}=A_{T}\left(x_{1}-T\right)^{2}+\mathrm{o}\left(\left(x_{1}-T\right)^{2}\right)$.

The function $T \mapsto A_{T}$ is the same as in Theorem 2.10.

4. If $T>t_{c c}$ then $\operatorname{Acc}_{S R}^{\alpha}(T)$ is open near $\gamma(T)$.

Figure 5 represents the evolution of $A c c_{S R}^{\alpha}(T)$ in function of $T$ in the plane $\left(x_{1}, x_{n}\right)$. It is open in a neighborhood of $\gamma(T)$ if $T>t_{c c}$, contrarily to the affine case where it becomes open only beyond $t_{c}$.

Remark 2.22. To compare the system (14) with its associated affine system (16) we need the following reparametrizing (see proof of Lem. 2.20):

$$
\frac{\mathrm{d} s}{\mathrm{~d} t}=v
$$

which only holds if $v$ does not vanish. This condition is satisfied when the control $(v, u)$ is in a $\alpha$-neighborhood in $\mathrm{L}^{\infty}$ metric of the abnormal reference control $(1,0)$, for in this case $v$ is close to 1 in $\mathrm{L}^{\infty}$. Hence using this method it is only possible to describe a constrained accessibility set, i.e. in a $\alpha$-neighborhood in $\mathrm{L}^{\infty}$ metric of the reference abnormal control. 


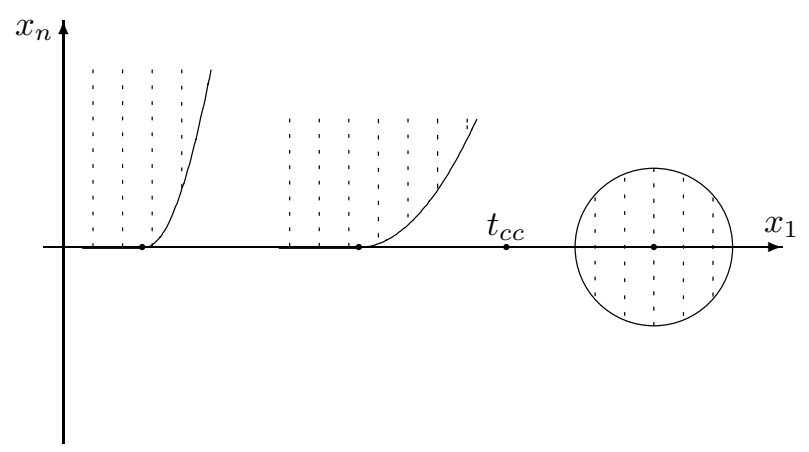

FIGURE 5

Proof of Theorem 2.21. The aim is to compare precisely systems (14) and (16) using Lemma 2.20 and to apply Theorem 2.10. In order to do this we first have to normalize the affine system (16) using Lemma 2.1. We denote by $A c c_{A}^{\beta}(T)$ the accessibility set at time $T$ with constraint $\beta$ of the affine system (16).

The system (7), $\dot{x}=f_{0}+u f_{1}$, is called normalized affine system and will be refered as (AN). Let $A c c_{A N}^{\eta}(T)$ denote the accessibility set at time $T$ for this system with the constraint: $|u| \leq \eta$. Due to the particular forms of the feedback $(\varphi, \alpha, \beta)$ and of the system, we have:

Lemma 2.23. $\varphi^{-1}\left(A c c_{A N}^{\eta}(T)\right)=A c c_{A}^{\beta}(T)$ where $\beta=\mathrm{O}(\eta)$ as $\eta \rightarrow 0$.

We know from Lemma 2.20 that:

$$
A c c_{S R}^{\alpha}(T) \subset \bigcup_{s \leq T} A c c_{A}^{\frac{\alpha}{1-\alpha}}(s)
$$

Hence in the normalized coordinates we get:

$$
\varphi\left(A c c_{S R}^{\alpha}(T)\right) \subset \bigcup_{s \leq T} A c c_{A N}^{\beta}(s)
$$

We shall use Theorem 2.10, which describes the boundary of $A c c_{A N}^{\beta}(s)$ in the plane $\left(x_{1}, x_{n}\right)$, to study the boundary of $\bigcup_{s \leq T} A c c_{A N}^{\beta}(s)$. Using the fact that $t$ the function $t \mapsto A_{T}$ is continuous and decreasing on [0,tc $[$, we can assert that the boundary of $\bigcup_{s \leq T} A c c_{A N}^{\beta}(s)$ is given, in the plane $\left(x_{1}, x_{n}\right)$, close to the point $(T, 0)$, by the following curve, see Figure 6:

- if $x_{1} \leq T$ then $x_{n}=0$

- if $x_{1} \geq T$ then $x_{n}=A_{T}\left(x_{1}-T\right)^{2}+\mathrm{o}\left(\left(x_{1}-T\right)^{2}\right)$.

Let $x_{n}=f\left(x_{1}\right)$ denote the curve parametrizing the boundary of $\varphi\left(\operatorname{Acc}_{S R}^{\alpha}(T)\right)$ in the plane $\left(x_{1}, x_{n}\right)$. From inclusion (17) we get:

- if $x_{1} \leq T$ then $f\left(x_{1}\right) \geq 0$;

- if $x_{1} \geq T$ then $f\left(x_{1}\right) \geq A_{T}\left(x_{1}-T\right)^{2}+\mathrm{o}\left(\left(x_{1}-T\right)^{2}\right)$.

Let us now prove the converse inequality. To this aim we shall use varying constraints depending on $x_{1}-T$. We proceed in the following way. From Lemma 2.23:

$$
\varphi^{-1}\left(A c c_{A N}^{\eta}\left(\frac{T}{\sqrt{1+\eta^{2}}}\right)\right) \subset A c c_{A}^{\beta}\left(\frac{T}{\sqrt{1+\eta^{2}}}\right)
$$




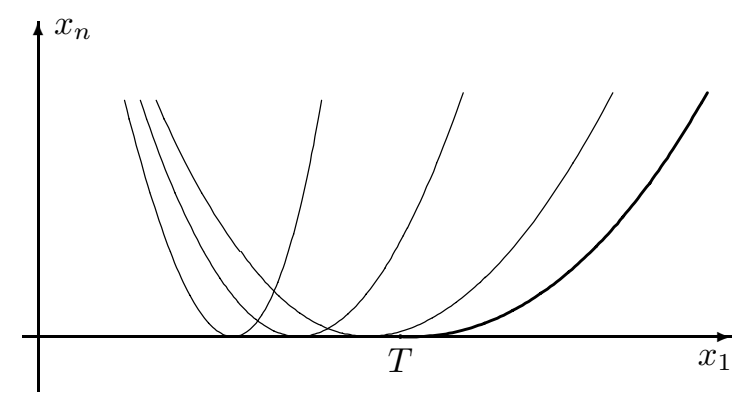

FIGURE 6

where $\beta=\mathrm{O}(\eta)$. Without loss of generality we can assume $\beta \leq \eta$, and thus:

$$
\operatorname{Acc}_{A}^{\beta}\left(\frac{T}{\sqrt{1+\eta^{2}}}\right) \subset \operatorname{Acc}_{A}^{\eta}\left(\frac{T}{\sqrt{1+\eta^{2}}}\right) .
$$

Now from Lemma 2.20, we get that for any $\eta>0$ small enough:

$$
\operatorname{Acc}_{A}^{\eta}\left(\frac{T}{\sqrt{1+\eta^{2}}}\right) \subset A c c_{S R}^{\alpha}(T) .
$$

And thus in the normalized coordinates:

$$
\operatorname{Acc}_{A N}^{\eta}\left(\frac{T}{\sqrt{1+\eta^{2}}}\right) \subset \varphi\left(A c c_{S R}^{\alpha}(T)\right) .
$$

Let $f_{A N}\left(x_{1}\right)$ be the function parametrizing the boundary of $A c c_{A N}^{\eta}\left(\frac{T}{\sqrt{1+\eta^{2}}}\right)$ in the plane $\left(x_{1}, x_{n}\right)$. We know from Theorem 2.10 that:

$$
f_{A N}\left(x_{1}\right)=A_{\frac{T}{\sqrt{1+\eta^{2}}}}\left(x_{1}-\frac{T}{\sqrt{1+\eta^{2}}}\right)^{2}+\mathrm{o}\left(\left(\frac{T}{\sqrt{1+\eta^{2}}}\right)^{2}\right)
$$

provided $x_{1}-\frac{T}{\sqrt{1+\eta^{2}}}=\mathrm{o}(\eta)$. This latter condition is fulfilled if $\eta=\left(x_{1}-T\right)^{\frac{3}{4}}$, and in this case we have moreover: $\eta^{2}=\mathrm{o}\left(x_{1}-T\right)$. On the other part, from the continuity of $t \mapsto A_{t}$ :

$$
A_{\frac{T}{\sqrt{1+\eta^{2}}}}=A_{T}+\mathrm{o}(1) \quad \text { as } x_{1} \rightarrow T .
$$

We obtain: $f_{A N}\left(x_{1}\right)=A_{T}\left(x_{1}-T\right)^{2}+\mathrm{o}\left(\left(x_{1}-T\right)^{2}\right)$. Finally, from inclusion (18) we conclude:

$$
f\left(x_{1}\right) \leq A_{T}\left(x_{1}-T\right)^{2}+\mathrm{o}\left(\left(x_{1}-T\right)^{2}\right)
$$

which ends the proof. 


\subsubsection{Splitting-up of the sphere near an abnormal direction}

Let $T>0$ small enough so that Properties 2 and 3 of Theorem 2.21 are satisfied. In particular the reference abnormal trajectory $\gamma$ is minimizing, see Section 3.1. Then $A=\gamma(T)$ belongs to the sub-Riemannian sphere $S(0, T)$ with radius $T$. If controls steering 0 to points of the boundary of $A c c_{S R}^{\alpha}(T)$ in $x_{n}>0$ (that are $\mathrm{L}^{\infty}$ optimal) are actually globally optimal, then this boundary is included in the sphere $S(0, T)$. In this case the sphere splits into two sectors near $\gamma(T)$, bordered by the first Pontryagin's cone $x_{n}=0$ :

- sector $x_{n}>0$ corresponding to the previous description;

- sector $x_{n}<0$.

According to the previous results, final points at time $T$ associated to controls which are $\mathrm{L}^{\infty}$-close to the reference abnormal control are in the first sector: $x_{n}>0$. Obviously due to controllability of the system the sector $x_{n}<0$ is accessible. In fact a basic calculus shows:

Lemma 2.24. For any neighborhood $V$ of the point $A$ in $\mathbb{R}^{n}$ we have:

$$
S(0, T) \cap V \cap\left(x_{n}<0\right) \neq \emptyset
$$

These points in $\left(x_{n}<0\right)$ are reached by controls which are close to the reference control in $\mathrm{L}^{2}$ metric but not in $\mathrm{L}^{\infty}$ metric. More precisely:

Lemma 2.25. Let $M_{n}=E_{T}\left(u_{n}\right) \in S(0, T)$ whose last coordinate $x_{n}$ is strictly negative. Let $u$ denote the abnormal reference control. We suppose that $M_{n}$ converges to $A=E(u)$ in $\mathbb{R}^{n}$. Then $u_{n}$ converges to $u$ in $\mathrm{L}^{2}([0, T])$ but not in $\mathrm{L}^{\infty}([0, T])$.

Hence near the abnormal direction the sphere is splits into two sectors:

- the $\mathrm{L}^{\infty}$-sector: $\left(x_{n}>0\right) \cap S(0, T)$ (described by Th. 2.21), made of end-points of minimizing trajectories associated to controls $\mathrm{L}^{\infty}$-close to the abnormal reference control;

- the $\mathrm{L}^{2}$-sector: $\left(x_{n}<0\right) \cap S(0, T)$, made of points reached by minimizing controls $\mathrm{L}^{2}$-close, but not $\mathrm{L}^{\infty}$-close to the abnormal reference control.

The contact of the first sector is known, but not the second one a priori. Anyway according to the Tangency theorem (see [23]), under some nice stratification assumptions, this $\mathrm{L}^{2}$-sector ramifies tangently to the Pontryagin cone $x_{n}=0$, see Figure 7 .

We summarize these results in the following theorem:

Theorem 2.26. Under the assumptions and with the notations of Theorem 2.21, if $T$ is small enough so that Properties 2 and 3 are satisfied, the sub-Riemannian sphere $S(0, T)$ splits into two sectors near $\gamma(T)$ :

1. the $\mathrm{L}^{\infty}$-sector: $\left(x_{n}>0\right) \cap S(0, T)$, made of end-points of minimizing trajectories associated to controls which are close to the abnormal reference control in $\mathrm{L}^{\infty}$-topology. Hence minimizing trajectories steering 0 to these points are close to $\gamma$ in $\mathrm{C}^{1}$-topology. Moreover in the plane $\left(x_{1}, x_{n}\right)$, its graph is:

$$
x_{1} \geq T, x_{n} \sim A_{T} \cdot\left(x_{1}-T\right)^{2}
$$

where $T \mapsto A_{T}$ is continuous, positive and decreasing;

2. the $\mathrm{L}^{2}$-sector: $\left(x_{n}<0\right) \cap S(0, T)$, made of end-points associated to minimizing controls which are close to the abnormal reference control in $\mathrm{L}^{2}$-topology, but not in $\mathrm{L}^{\infty}$-topology. Hence trajectories steering 0 to these points are close to $\gamma$ in $\mathrm{C}^{0}$-topology, but not in $\mathrm{C}^{1}$-topology. This sector is tangent to the abnormal direction.

These two sectors are separated by the first Pontryagin's cone $x_{n}=0$ along $\gamma$ (see Fig. 7). 


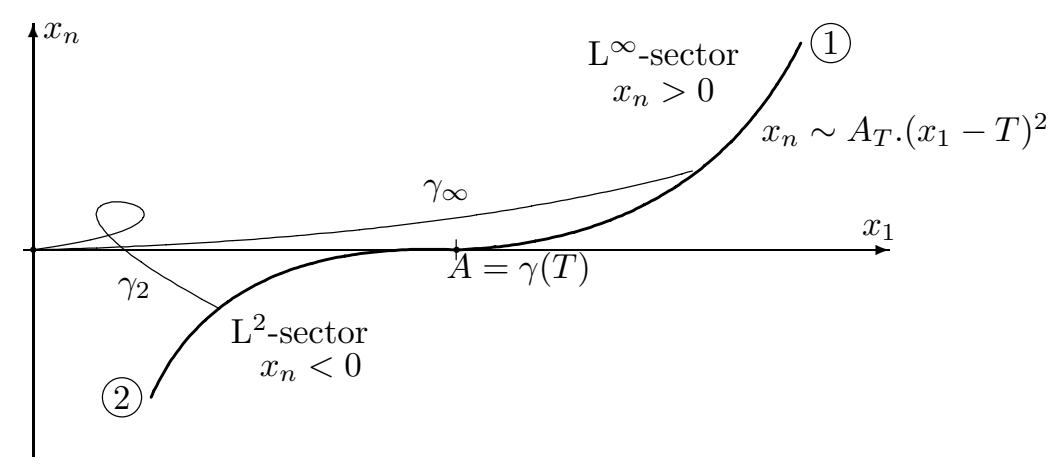

FiguRe 7

Typical example: the Martinet case. Consider the two following vector fields in $\mathbb{R}^{3}$ :

$$
X=\frac{\partial}{\partial x}+\frac{y^{2}}{2} \frac{\partial}{\partial z}, Y=\frac{\partial}{\partial y}
$$

and endow the distribution spanned by these vector fields with an analytic metric $g$ of the type:

$$
g=a d x^{2}+c d y^{2}
$$

where $a=(1+\alpha y)^{2}$ and $c=(1+\beta x+\gamma y)^{2}$. The abnormal reference control for the sub-Riemannian system $\dot{x}=v X(x)+u Y(x)$ with constraint $v^{2}+u^{2} \leq 1$ is $v=1, u=0$, and corresponds to the trajectory $\gamma$ : $x(t)=t, y(t)=z(t)=0$. We have, see [11,24]:

Lemma 2.27. Assumptions $\left(H_{0}-H_{3}\right)$ are fulfilled along $\gamma$ if and only if $\alpha \neq 0$. In this case branches 1 and 2 (see Fig. 7 with $x_{1}=x, x_{n}=z$ ) have the following contacts with the abnormal direction:

- branch 1: $x \geq T, z=\frac{1}{2 T \alpha^{2}}(x-T)^{2}+\mathrm{o}\left((x-T)^{2}\right)$;

- branch $2: x \leq T, z \sim \frac{1}{6}(1+\mathrm{O}(T))(x-T)^{3}$.

Remark 2.28. The coefficient $A_{T}$ of the first branch can be computed directly or using formula (10) (see Rem. 2.11).

As we are in dimension 3 , results of Theorem 2.21 are in fact available on $\mathbb{R}^{+}$, see Remark 2.8. The $\mathrm{L}^{2}$-sector is $z<0$ and the $\mathrm{L}^{\infty}$-sector is $z>0$.

Proof of Lemma 2.24. Consider the sub-Riemannian system (14) in the coordinates $\left(x_{1}, \ldots, x_{n}\right)$ of Theorem 2.21:

$$
\begin{aligned}
\dot{x}_{1} & =v\left(1+x_{2}+x_{n} f_{1}\left(x_{1}\right)+Z_{1}\right) \\
\dot{x}_{2} & =v\left(x_{3}+x_{n} f_{2}\left(x_{1}\right)+Z_{2}\right) \\
& \vdots \\
\dot{x}_{n-1} & =u+x_{n} f_{n-1}\left(x_{1}\right)+Z_{n-1} \\
\dot{x}_{n} & =v \sum_{i=2}^{n} a_{i j}\left(x_{1}\right) x_{i} x_{j}+Z_{n}
\end{aligned}
$$

where the 1 -jet (resp. the 2 -jet) of $Z_{1}, \ldots, Z_{n-1}$ (resp. $Z_{n}$ ) along $u=0$ is equal to 0 . 
Our goal is to construct a control close to the reference abnormal control in $\mathrm{L}^{2}$ metric, whose associated final point at time $T$ is close to $(T, 0, \ldots, 0)$ and is such that $x_{n}<0$. Let $\varepsilon>0$. Consider the following control $v$ (see Fig. 8):

- if $0 \leq t \leq \frac{T-\varepsilon}{2}$ then $v(t)=1$;

- if $\frac{T-\varepsilon}{2} \leq t \leq \frac{T+\varepsilon}{2}$ then $v(t)=-1$;

- if $\frac{T+\varepsilon}{2} \leq t \leq T$ then $v(t)=1$.

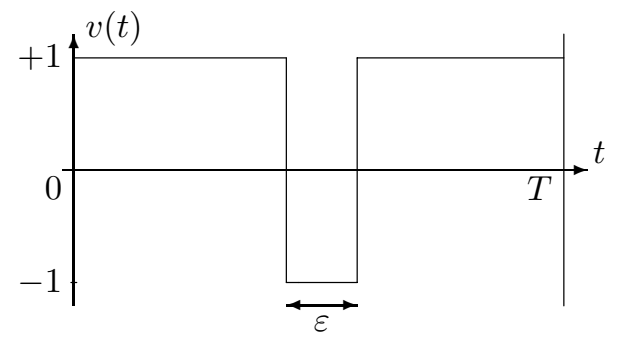

Figure 8

Set $u=0$. It is clear that $(v, u)$ is abnormal (but not minimizing). Consider the following perturbation (see Fig. 9):

- if $0 \leq t \leq \frac{T-\varepsilon}{2}$ then $\delta v(t)=\delta u(t)=0$;

- if $\frac{T-\varepsilon}{2}<t \leq \frac{T}{2}$ then $\delta v(t)=1-\sqrt{1-\varepsilon^{2}}, \delta u(t)=\varepsilon$;

- if $\frac{T}{2}<t \leq \frac{T+\varepsilon}{2}$ then $\delta v(t)=1-\sqrt{1-\varepsilon^{2}}, \delta u(t)=-\varepsilon$;

- if $\frac{T+\varepsilon}{2}<t \leq T$ then $\delta v(t)=\delta u(t)=0$.

It is clear that $(v+\delta v)^{2}+(\delta u)^{2}=1$. Moreover: $\|\delta v\|_{\mathrm{L}^{1}}=\mathrm{O}\left(\varepsilon^{3}\right)$ and $\|\delta u\|_{\mathrm{L}^{1}}=\mathrm{O}\left(\varepsilon^{2}\right)$.
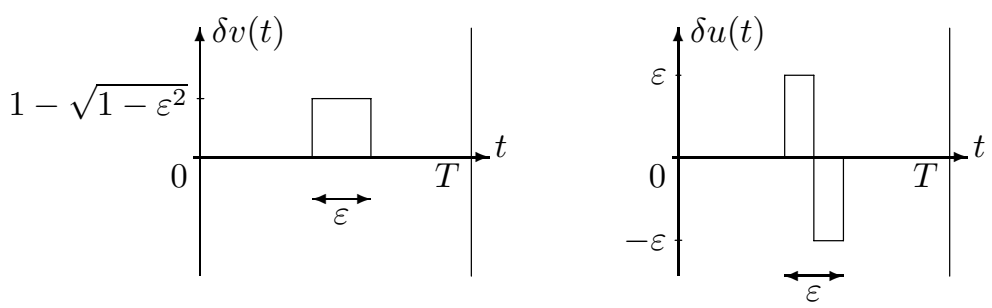

Figure 9

The end-point mapping $E_{T}$ being $\mathrm{C}^{\infty}$ in $\mathrm{L}^{1}$ topology (see for instance [17]), we have:

$$
\forall i \in\{1, \ldots, n-1\} \quad E_{T}^{i}(v+\delta v, \delta u)=E_{T}^{i}(v, 0)+d E_{T}^{i}(v, 0) \cdot(\delta v, \delta u)+\mathrm{O}\left(\varepsilon^{4}\right)
$$

and

$$
E_{T}^{n}(v+\delta v, \delta u)=E_{T}^{n}(v, 0)+d E_{T}^{n}(v, 0) \cdot(\delta v, \delta u)+\frac{1}{2} d^{2} E_{T}^{n}(v, 0) \cdot(\delta v, \delta u)^{[2]}+\mathrm{O}\left(\varepsilon^{6}\right)
$$


Moreover we have $E_{T}^{i}(v, 0)=\delta_{i}^{1}(T-\varepsilon)$ for $i=1, \ldots, n$. On the other part the linearized system along $(v, u)$ is:

$$
\dot{y}_{1}=\delta v+v y_{2}, \dot{y}_{2}=v y_{3}, \ldots, \dot{y}_{n-2}=v y_{n-1}, \dot{y}_{n-1}=\delta u, \dot{y}_{n}=0 .
$$

Let us calculate $y_{n-1}(t)$ :

- if $0 \leq t \leq \frac{T-\varepsilon}{2}$ then $y_{n-1}(t)=0$;

- if $\frac{T-\varepsilon}{2}<t \leq \frac{T}{2}$ then $y_{n-1}(t)=\varepsilon\left(t-\frac{T-\varepsilon}{2}\right)$;

- if $\frac{T}{2}<t \leq \frac{T+\varepsilon}{2}$ then $y_{n-1}(t)=-\varepsilon\left(t-\frac{T+\varepsilon}{2}\right)$;

- if $\frac{T+\varepsilon}{2}<t \leq T$ then $y_{n-1}(t)=0$.

In particular: $\left\|y_{n-1}\right\|_{\mathrm{L}^{\infty}}=\mathrm{O}\left(\varepsilon^{2}\right)$, and $y_{n-1}$ is equal to 0 outside an interval of length $\varepsilon$. We get easily:

$$
\left\|y_{n-2}\right\|_{L^{\infty}}=\cdots=\left\|y_{1}\right\|_{L^{\infty}}=\mathrm{O}\left(\varepsilon^{3}\right) .
$$

Hence using (19):

$$
\begin{aligned}
E_{1}^{T}(v+\delta v, \delta u) & =T-\varepsilon+\mathrm{O}\left(\varepsilon^{3}\right) \\
E_{2}^{T}(v+\delta v, \delta u) & =\mathrm{O}\left(\varepsilon^{3}\right) \\
& \vdots \\
E_{n-2}^{T}(v+\delta v, \delta u) & =\mathrm{O}\left(\varepsilon^{3}\right) \\
E_{n-1}^{T}(v+\delta v, \delta u) & =\mathrm{O}\left(\varepsilon^{2}\right) .
\end{aligned}
$$

Let us now compute the last coordinate. We have to calculate $z_{n}$, where:

$$
\dot{z}_{n}=v \sum_{i, j=2}^{n-1} a_{i j}(t) y_{i} y_{j}
$$

It represents the intrinsic second-order derivative. From (21) we get:

$$
\dot{z}_{n}(t)=v(t) a_{n-1, n-1}(t) y_{n-1}^{2}(t)+y_{n-1}(t) \mathrm{O}\left(\varepsilon^{3}\right)+\mathrm{O}\left(\varepsilon^{6}\right) .
$$

As $y_{n-1}$ is equal to 0 outside $\left[\frac{T-\varepsilon}{2}, \frac{T+\varepsilon}{2}\right]$, we have:

$$
\int_{0}^{T} y_{n-1}(t) \mathrm{O}\left(\varepsilon^{3}\right) \mathrm{d} t=\mathrm{O}\left(\varepsilon^{6}\right)
$$

Moreover the coefficient $a_{n-1, n-1}$ is continuous and does not vanish on $[0, T]$, hence there exists $\alpha>0$ such that $a_{n-1, n-1}(t) \geq \alpha$ on $[0, T]$. We get:

$$
z_{n}(T) \leq-\alpha \int_{\frac{T-\varepsilon}{2}}^{\frac{T+\varepsilon}{2}} y_{n-1}^{2}(t) \mathrm{d} t+\mathrm{O}\left(\varepsilon^{6}\right) \leq-\frac{\alpha \varepsilon^{5}}{12}+\mathrm{O}\left(\varepsilon^{6}\right) .
$$

Therefore from (20):

$$
E_{n}^{T}(v+\delta v, \delta u) \leq-\frac{\alpha \varepsilon^{5}}{12}+\mathrm{O}\left(\varepsilon^{6}\right) .
$$

Hence for any neighborhood $V$ of $(T, 0, \ldots, 0), A c c(T) \cap V$ contains points such that $x_{n}<0$, and hence the same goes for $S(0, T) \cap V$ since the abnormal reference trajectory is minimizing. By construction, controls steering to these points are $\mathrm{L}^{2}$-close but not $\mathrm{L}^{\infty}$-close to the reference abnormal control. 
Proof of Lemma 2.25. As $M_{n}$ belongs to the sphere $S(0, T)$, we have: $\left\|u_{n}\right\|_{\mathrm{L}^{2}}=\|u\|_{\mathrm{L}^{2}}=T$, thus the sequence $\left(u_{n}\right)_{n \in \mathbb{N}}$ is bounded in $\mathrm{L}^{2}$. Hence up to a subsequence we can assume that $u_{n}$ converges weakly to $v \in \mathrm{L}^{2}$ (denoted by $u_{n} \hookrightarrow v$ ). From the continuity of the end-point mapping $E_{T}$ in the weak topology on $\mathrm{L}^{2}$ (see [23]), we can assert that $M_{n}=E_{T}\left(u_{n}\right)$ converges to $E_{T}(v)$, and thus $E_{T}(v)=E_{T}(u)$. The assumptions on the reference abnormal trajectory imply that $v=u$. Hence $u_{n} \hookrightarrow u$, and on the other part : $\left\|u_{n}\right\|_{\mathrm{L}^{2}}=\|u\|_{\mathrm{L}^{2}}$, therefore $u_{n}$ converges (strongly) towards $u$ in $\mathrm{L}^{2}$.

Moreover results stated by Theorem 2.21 imply that $u_{n}$ does not converge towards $u$ in $L^{\infty}$ (because it would imply that $x_{n} \geq 0$ ).

\section{Application: Optimality of abnormal trajectories}

In this section we apply our previous theory on accessibility sets to studying optimality of abnormal trajectories; this leads us to find again some well-known results. Indeed in the notations of Theorem 2.10, this theorem implies in particular that if $T<t_{c c}$ then $\gamma$ is isolated in $\mathrm{C}^{0}$-topology in the space of all trajectories which connect given end-points, and thus is optimal for any cost in this topology. This well-known property, called rigidity, was intensively studied. The main results concerning this analysis in a generic context were given first in [22] and [9] for single-input affine systems, then in [4,14,18,25], for sub-Riemannian systems, and in [6] in general. Moreover these authors developed a Morse theory in order to characterize conjugate points, that is, points beyond which the abnormal trajectory is no more optimal.

Hence results given in this section are not really new. However they are slightly different from the results cited above. Indeed on the one part in [9] were obtained necessary and sufficient conditions for $\mathrm{C}^{0}$-time-optimality of abnormal trajectories of single-input affine systems without any constraint on the control. Here we improve their statement by adding a constraint on the control and studying the problem of minimizing any cost. On the other part, in [6] was made a general theory (i.e. for nonlinear systems) on optimality of abnormal trajectories in $\mathrm{L}^{\infty}$ topology on the controls. Results given here are valid in the $\mathrm{C}^{0}$-topology on the trajectories (but only for single-input affine systems). Moreover we study the equivalence between the time-optimality problem and the problem of minimizing any cost, the final time being fixed or not. Finally Theorem 3.10, which concerns optimality of abnormal trajectories for sub-Riemannian systems of rank 2, makes a link between the works of [9] and $[4,5]$.

\subsection{Optimality of abnormal trajectories for single-input affine systems}

Consider the single-input affine system (5) with constraint (6), and suppose assumptions $\left(H_{0}-H_{4}\right)$ are fulfilled along a reference abnormal trajectory $\gamma$. We first study the time-optimal problem, and then the problem of minimizing some cost.

\subsubsection{Time optimality}

Definition 3.1. $\quad$ - The trajectory $\gamma$ is said $\mathrm{C}^{0}$-time-minimal on $[0, T]$ if there exists a $\mathrm{C}^{0}$-neighborhood of $\gamma$ such that $T$ is the minimal time to steer $\gamma(0)$ to $\gamma(T)$ among the solutions of the system (5) with the constraint (6) that are entirely contained in this neighborhood.

- Recall that $\gamma$ is associated to the control $u=0$. Let $\delta>0$. The trajectory $\gamma$ is said $\mathrm{L}^{\infty}$-time-minimal on $[0, T]$ if there exists a neighborhood of 0 in $\mathrm{L}^{\infty}([0, T+\delta])$ such that $T$ is the minimal time to steer $\gamma(0)$ to $\gamma(T)$ among trajectories associated to controls of this neighborhood.

Obviously if $\gamma$ is $\mathrm{C}^{0}$-time-minimal then it is $\mathrm{L}^{\infty}$-time-minimal.

Theorem 3.2. Under assumptions of Theorem 2.10, the trajectory $\gamma$ is $\mathrm{C}^{0}$-time-minimal if and only if $T<t_{c c}$. Moreover $\gamma$ is not $\mathrm{L}^{\infty}$-time-minimal if $T>t_{c c}$.

The proof is clear by inspecting Figure 2 and proof of Theorem 2.10.

Remark 3.3. If $n=3$, we have $t_{c c}=+\infty$ provided assumptions $\left(H_{0}-H_{3}\right)$ are fulfilled on $\mathbb{R}^{+}$. Hence in this case $\gamma$ is $\mathrm{C}^{0}$-time-minimal on $\mathbb{R}^{+}$. 


\subsubsection{Optimality for some cost}

Let us now consider the problem of minimizing some cost $C(T, u)$, also denoted by $C_{T}(u)$, where $C$ is a smooth function satisfying the following additional assumption along the reference singular trajectory $\gamma$ :

$\left(H_{5}\right) \forall T \quad \operatorname{rank}\left(d E_{T}(0), d C_{T}(0)\right)=n$

i.e. the singularity of the end-point mapping of the extended system has codimension 1, and in particular the cost is independant from the end-point mapping along $\gamma$. We consider several optimization problems:

1. final time not fixed: the aim is to steer the system from $x_{0}$ to $x_{1}$ in some time $T$ (not preassigned) and minimizing the cost $C$;

2. final time fixed: let $T>0$ fixed; the aim is to steer the system from $x_{0}$ to $x_{1}$ in time $T$ and minimizing the cost $C_{T}$.

1. Final time not fixed.

Definition 3.4. - The trajectory $\gamma$ is said to be $\mathrm{C}^{0}$-cost-minimal on $[0, T]$ if there exists a $\mathrm{C}^{0}$-neighborhood of $\gamma$ such that for any trajectory $q$ contained in this neighborhood, with $q(0)=\gamma(0)$ and $q(t)=\gamma(T)$, we have: $C(t, v) \geq C(T, 0)$, where $v$ is the control associated to $q$.

- Let $\delta>0$. The trajectory $\gamma$ is said to be $\mathrm{L}^{\infty}$-cost-minimal on $[0, T]$ if there exists a neighborhood of 0 in $\mathrm{L}^{\infty}([0, T+\delta])$ such that, for any trajectory $q$ associated to a control $v$ of this neighborhood, with $q(0)=\gamma(0)$ and $q(t)=\gamma(T)$, we have: $C(t, v) \geq C(T, 0)$.

Obviously the $\mathrm{C}^{0}$-cost-minimality implies the $\mathrm{L}^{\infty}$-cost-minimality.

We have the following result (compare with [6]):

Theorem 3.5. Under assumptions $\left(H_{0}-H_{5}\right)$, the trajectory $\gamma$ is $\mathrm{C}^{0}$-cost-minimal if and only if it is $\mathrm{C}^{0}$-timeminimal. Actually, $\gamma$ is $\mathrm{C}^{0}$-cost-minimal if $T<t_{c c}$, and is not $\mathrm{L}^{\infty}$-cost-minimal if $T>t_{c c}$.

Hence if the final time is not fixed then both problems of cost-minimization and time-minimization are equivalent.

Proof. If $T<t_{c c}$, then the abnormal trajectory $\gamma$ is isolated in a $\mathrm{C}^{0}$-neighborhood, hence in particular is $\mathrm{C}^{0}$ cost-minimal. If $T>t_{c c}$, we know that $\gamma$ is no more time-minimal since the point $(T, 0, \ldots, 0)$ belongs to $A c c(t)$ for some $t<T$, see Figure 10 .

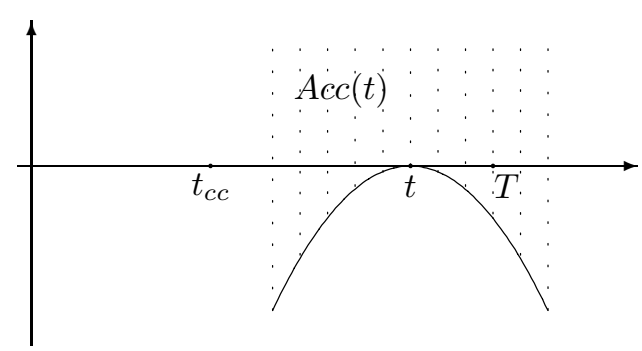

Figure 10

To prove that $\gamma$ is no more $\mathrm{L}^{\infty}$-cost-minimal, we have to show that the point $(T, 0, \ldots, 0)$ belongs to $M_{r}(t)$ for some $r<C_{T}(0)$, where $C_{T}(0)$ is the cost of $\gamma$ at time $T$, and $M_{r}(t)$ is the level set $r$ at time $t$ of the value function associated to the cost $C$.

In the coordinates $\left(x_{1}, \ldots, x_{n}\right)$ of Lemma 2.1 , the end-point mapping is $E_{t}=\left(E_{1}^{t}, \ldots, E_{n}^{t}\right)$, where:

$$
\operatorname{rank}\left(d E_{1}^{t}(0), \ldots, d E_{n}^{t}(0)\right)=n-1 \quad \text { and } \quad d E_{n}^{t}(0)=0 .
$$


Instead of working in $\mathrm{L}^{2}([0, T])$, we choose as control space the Sobolev space $\mathrm{H}^{1}([0, T])=\left\{f \in \mathrm{L}^{2}([0, T]) / f^{\prime} \in\right.$ $\left.\mathrm{L}^{2}([0, T])\right\}$. Endowed with the norm $\|f\|_{\mathrm{H}^{1}}=\sqrt{\|f\|_{\mathrm{L}^{2}}^{2}+\left\|f^{\prime}\right\|_{\mathrm{L}^{2}}^{2}}$, this is a Hilbert space. The reason to use it is the following: it can be immersed in a compact way in $\mathrm{C}^{0}([0, T])$, and in the proof we shall need continuous controls.

The end-point mapping is still differentiable in $\mathrm{H}^{1}$ since it is differentiable in $\mathrm{L}^{2}$. From assumption $\left(H_{4}\right)$, the end-point mapping is independant from the $\operatorname{cost}$ along $\gamma$, hence at time $T$ there exist $n$ independant vector fields $e_{0}^{T}, e_{1}^{T}, \ldots, e_{n-1}^{T}$ such that:

$$
d C_{T}(0) \cdot e_{0}^{T}=1 \quad \text { and } \quad d E_{i}^{T}(0) \cdot e_{i}^{T}=1, i=1 \ldots n-1 .
$$

We can decompose $\mathrm{H}^{1}$ in the following way:

$$
\mathrm{H}^{1}([0, T])=\mathbb{R} e_{1}^{T} \oplus \mathbb{R} e_{2}^{T} \oplus \cdots \oplus \mathbb{R} e_{n-1}^{T} \oplus \mathbb{R} e_{0}^{T} \oplus F
$$

where ker $d E_{i}^{T}(0)=\bigoplus_{j \neq i} \mathbb{R} e_{j}^{T} \oplus F$ and ker $d C_{T}(0)=\bigoplus_{j \neq 0} \mathbb{R} e_{j}^{T} \oplus F$.

Let $Q_{T}(u)=d^{2} E_{n}^{T}(0) .(u, u)$ (as in Lem. 2.15). The kernel of the first derivative is: $\operatorname{ker} d E_{T}(0)=\mathbb{R} e_{0}^{T} \oplus F$, and hence the intrinsic second-order derivative at time $T$ along $\gamma$ is the quadratic form $Q_{T / \mathbb{R} e_{0}^{T} \oplus F}$. On the other part the space $\mathbb{R} e_{1}^{T} \oplus \mathbb{R} e_{0}^{T} \oplus F$ represents the domain of the latter quadratic form where the limit condition on the first coordinate has been relaxed, hence it is the domain of the reduced operator $D_{1}$. Now by definition of the first conjugate times $t_{c c}$ and $t_{c}$, we have:

- if $T<t_{c c}: Q_{T} / \mathbb{R} e_{1}^{T} \oplus \mathbb{R}_{0}^{T} \oplus F$ is positive definite;

- if $t_{c c}<T<t_{c}: Q_{T / \mathbb{R} e_{0}^{T} \oplus F}$ is positive definite, and $Q_{T / \mathbb{R} e_{1}^{T} \oplus \mathbb{R}_{0}^{T} \oplus F}$ is indefinite;

- if $T>t_{c}: Q_{T / \mathbb{R} e_{0}^{T} \oplus F}$ is indefinite.

From now on we assume that $T>t_{c c}$. Then there exist $v, w$ in $\mathbb{R} e_{0}^{T} \oplus F$ such that $Q_{T}\left(e_{1}^{T}+v\right)<0$ and $Q_{T}(w)>0$.

Remark 3.6. Note that we do not have necessarily $Q_{T}\left(e_{1}^{T}\right)<0$.

Let $\delta>0$ small. As $v$ and $w$ are continuous, it is possible to extend them on $[T, T+\delta]$ respectively by $v(T)$ and $w(T)$ (here is the role of $\mathrm{H}^{1}$ ). Consider now the following control $u \in \mathrm{H}^{1}([0, T])$ :

$$
u=a_{1} e_{1}^{T}+a_{2} e_{2}^{T}+\cdots+a_{n-1} e_{n-1}^{T}+a_{1} v+a_{n} w
$$

where $a_{1}, \ldots, a_{n}$ are real numbers. In the same way we can extend $u$ on $[0, T+\delta]$. We shall prove that we can choose $a_{1}, \ldots, a_{n}$ small and $t$ close to $T$ such that the previous control $u$ satisfies:

$$
|u| \leq \eta, t \leq T+\delta, E_{t}(u)=\gamma(T), C_{t}(u)<C_{T}(0)
$$

i.e. $\gamma(T)$ belongs to $M_{r}(t)$ for some $r<C_{T}(0)$. To this aim we shall first use the Implicit Function theorem on $a_{1}, \ldots, a_{n}$ (to take into account the $n-1$ first coordinates, i.e. the controllable part of the system), then the Mean Value theorem on the last coordinate, and endly choose $t$ in order to make the cost lower than $C_{T}(0)$. Note that in this method all reasonings are in finite dimension.

Let us expand, for $t$ close to $T$ and $i=1, \ldots, n-1$ :

$$
E_{i}^{t}(u)=E_{i}^{t}(0)+d E_{i}^{t}(0) \cdot u+\mathrm{o}\left(\|u\|_{\mathrm{H}^{1}([0, t])}\right) .
$$

Moreover:

- $E_{1}^{t}(0)=t$, and for $i=2, \ldots, n-1: E_{i}^{t}(0)=0$;

- $\mathrm{o}\left(\|u\|_{\mathrm{H}^{1}([0, t])}\right)=\mathrm{o}\left(\|u\|_{\mathrm{H}^{1}([0, T+\delta])}\right)=\mathrm{o}\left(a_{1}, \ldots, a_{n}\right)$; 
- for $i=1, \ldots, n-1: d E_{i}^{t}(0)=d E_{i}^{T}(0)+\mathrm{O}(t-T)$.

Therefore we get, for $i=1, \ldots, n-1$ :

$$
d E_{i}^{t}(0) \cdot u=a_{i}+\mathrm{o}\left(a_{1}, \ldots, a_{n}, t-T\right)
$$

and thus:

$$
E_{t}(u)=\left(t+a_{1}+\mathrm{o}\left(a_{1}, \ldots, a_{n}, t-T\right), a_{2}+\mathrm{o}\left(a_{1}, \ldots, a_{n}, t-T\right), \ldots, a_{n-1}+\mathrm{o}\left(a_{1}, \ldots, a_{n}, t-T\right), E_{n}^{t}(u)\right) .
$$

Let us first solve the system of equations:

$$
E_{1}^{t}(u)=T, E_{2}^{t}(u)=\cdots=E_{n-1}^{t}(u)=0 .
$$

We get from the Implicit Function theorem:

$$
\forall i \in\{1, \ldots, n-1\} \quad a_{i}=f_{i}\left(T-t, a_{n}\right)
$$

and moreover:

$$
a_{1}=T-t+\mathrm{o}\left(T-t, a_{n}\right) \text { and for } i=2, \ldots, n-1: a_{i}=\mathrm{o}\left(T-t, a_{n}\right) .
$$

Let us now calculate the last coordinate:

$$
E_{n}^{t}(u)=E_{n}^{t}(0)+d E_{n}^{t}(0) \cdot u+\frac{1}{2} d^{2} E_{n}^{t}(0) \cdot(u, u)+\mathrm{o}\left(\|u\|_{\mathrm{H}^{1}}^{2}\right)=Q_{t}(u)+\mathrm{o}\left((T-t)^{2}, a_{n}^{2}\right) .
$$

Moreover:

$$
Q_{t}(u)=Q_{t}\left(a_{1}\left(e_{1}^{T}+v\right)+a_{2} e_{2}^{T}+\cdots+a_{n-1} e_{n-1}^{T}+a_{n} w\right)
$$

Hence

$$
E_{n}^{t}(u)=(T-t)^{2} Q_{t}\left(e_{1}^{T}+v\right)+a_{n}^{2} Q_{t}(w)+a_{n}(T-t) \check{Q}_{t}\left(e_{1}^{T}+v, w\right)+o\left((T-t)^{2}, a_{n}^{2}\right)=g\left(t, a_{n}\right)
$$

where $\check{Q}$ denotes the symmetric bilinear form associated to the quadratic form $Q$. On the one part: $g\left(T, a_{n}\right)=$ $a_{n}^{2} Q_{T}(w)+\mathrm{o}\left(a_{n}^{2}\right)>0$ if $a_{n}$ is small enough (by definition of $w$ ). On the other part: $g(t, 0)=(T-t)^{2} Q_{t}\left(e_{1}^{T}+\right.$ $v)+\mathrm{o}\left((T-t)^{2}\right)$. From the definition of $v$, we have: $Q_{T}\left(e_{1}^{T}+v\right)<0$. Hence if $t$ is close enough to $T$, but not equal to $T$, we get: $g(t, 0)<0$. Now applying the Mean Value theorem to $g$ we can assert that there exist $t$ close to $\mathrm{T}$ and $a_{n}$ small such that $g\left(t, a_{n}\right)=0$, that is $E_{n}^{t}(u)=0$. Actually there exist four such couples $\left(t, a_{n}\right)$ with $t>0$ or $t<0$, and $a_{n}>0$ or $a_{n}<0$.

Remark 3.7. For such a couple $\left(t, a_{n}\right)$ we can say more about the asymptotics of $t$ with respect to $a_{n}$, which will be useful in the sequel. To this aim let us solve the following equation, where $a_{n}$ is fixed:

$$
g\left(t, a_{n}\right)=0 .
$$

We have:

- $Q_{t}\left(e_{1}^{T}+v\right)=Q_{T}\left(e_{1}^{T}+v\right)+\mathrm{O}(T-t)$;

- $Q_{t}(w)=Q_{T}(w)+\mathrm{O}(T-t)$;

- $\check{Q}_{t}\left(e_{1}^{T}+v, w\right)=\check{Q}_{T}\left(e_{1}^{T}+v, w\right)+\mathrm{O}(T-t)$. 
Hence equation (22) becomes:

$$
Q_{T}\left(e_{1}^{T}+v\right)(T-t)^{2}+a_{n} \check{Q}_{T}\left(e_{1}^{T}+v, w\right)(T-t)+a_{n}^{2} Q_{T}(w)+\mathrm{o}\left((T-t)^{2}, a_{n}^{2}\right)=0 .
$$

If $a_{n}$ is fixed, close to 0 , we easily find two solutions $t_{1}<T$ and $t_{2}>T$ such that both satisfy:

$$
T-t=\beta a_{n}+\mathrm{o}\left(a_{n}\right), \beta \in \mathbb{R} \backslash\{0\} .
$$

To end the proof we have to study the cost $C_{t}(u)$ and prove that $t$ and $a_{n}$ can be chosen so that this cost is lower than $C_{T}(0)$. Recall vectors $v, w \in \mathbb{R} e_{0}^{T} \oplus F$ previously defined so that:

$$
Q_{T}\left(e_{1}^{T}+v\right)<0 \quad \text { and } \quad Q_{T}(w)>0
$$

We can write:

$$
v=\lambda e_{0}^{T}+v_{1}, w=\mu e_{0}^{T}+w_{1} \quad \text { where } v_{1}, w_{1} \in F .
$$

Up to adding $\varepsilon e_{0}^{T}, \varepsilon$ small, we can assume that $\mu \neq 0$. This does not change anything in inequalities (23), nor in our previous reasoning. Let us denote $C(t, u)=C_{t}(u)$. We can expand:

$$
C_{t}(u)=C_{T}(0)+\alpha(t-T)+\frac{\partial C}{\partial u}(T, 0) \cdot u+o(T-t,\|u\|)
$$

where $\alpha=\frac{\partial C}{\partial t}(T, 0)$. Moreover by definition of $u$ and $e_{i}^{T}$ :

$$
\frac{\partial C}{\partial u}(T, 0) \cdot u=d C_{T}(0) \cdot u=a_{1} \lambda+a_{n} \mu
$$

On the one part: $a_{1}=T-t+\mathrm{o}\left(T-t, a_{n}\right)$. On the other part from Remark 3.7 there exists $\beta \neq 0$ such that $a_{n}=\beta(t-T)+\mathrm{o}(t-T)$. Hence we obtain:

$$
C_{t}(u)=C_{T}(0)+(t-T)(\alpha-\lambda+\beta \mu)+\mathrm{o}(t-T)
$$

Now among the four solutions of Remark 3.7, we can choose $t$ and $a_{n}$ such that $\alpha-\lambda+\beta \mu \neq 0$ and $(t-T)(\alpha-$ $\lambda+\beta \mu)<0$. In these conditions:

which ends the proof.

$$
C_{t}(u)<C_{T}(0)
$$

2. Final time fixed.

Definition 3.8. - The trajectory $\gamma$ is said to be $\mathrm{C}^{0}$-cost-minimal on $[0, T]$ if there exists a $\mathrm{C}^{0}$-neighborhood of $\gamma$ such that for any trajectory $q$ contained in this neighborhood, with $q(0)=\gamma(0)$ and $q(T)=\gamma(T)$, we have: $C_{T}(v) \geq C_{T}(0)$, where $v$ is the control associated to $q$.

- The trajectory $\gamma$ is said to be $\mathrm{L}^{\infty}$-cost-minimal on $[0, T]$ if there exists a neighborhood of 0 in $\mathrm{L}^{\infty}([0, T])$ such that, for any trajectory $q$ associated to a control $v$ of this neighborhood, with $q(0)=\gamma(0)$ and $q(T)=\gamma(T)$, we have: $C_{T}(v) \geq C_{T}(0)$.

We have the following:

Theorem 3.9. The trajectory $\gamma$ is $\mathrm{C}^{0}$-cost-minimal if and only if $T<t_{c}$. Moreover, $\gamma$ is not $\mathrm{L}^{\infty}$-cost-minimal if $T>t_{c}$ (whereas $\gamma$ is $\mathrm{C}^{0}$-time-minimal if and only if $T<t_{c c}$ ), where $t_{c c}$ and $t_{c}$ denote the two types of first conjugate times of $\gamma$.

Hence in this case, the times at which $\gamma$ ceases to be minimizing are different in the time-optimal problem and cost-optimal problem: $\gamma$ ceases to be $\mathrm{C}^{0}$-time-optimal before it ceases to be $\mathrm{C}^{0}$-cost-optimal (since $t_{c c}<t_{c}$, see Lem. 2.7). 
Proof. The proof is quite similar to proof of Theorem 3.5 (but simpler) and is only sketched. In the coordinates $\left(x_{1}, \ldots, x_{n}\right)$ of Lemma 2.1 , we write $E_{T}=\left(E_{1}^{T}, \ldots, E_{n}^{T}\right)$, where $d^{2} E_{n}^{T}(0)=0$, and $d E_{1}^{T}(0), \ldots$, $d E_{n-1}^{T}(0), d C_{T}(0)$ are independant. We decompose:

$$
\mathrm{L}^{2}([0, T])=\mathbb{R} e_{1} \oplus \cdots \oplus \mathbb{R} e_{n-1} \oplus \mathbb{R} e_{0} \oplus F
$$

where $d E_{i}^{T}(0) \cdot e_{i}=1$ and $d C_{T}(0) \cdot e_{0}=1$. By definition of $t_{c}$ :

- if $T<t_{c}: Q_{/ \mathbb{R} e_{0} \oplus F}$ is positive definite;

- if $T>t_{c}: Q / \mathbb{R} e_{0} \oplus F$ is indefinite;

where $Q=d^{2} E_{n}^{T}(0)$. Hence if $T>t_{c}$ there exist $v, w \in \mathbb{R} e_{0} \oplus F$ such that $Q(v)>0$ and $Q(w)<0$. Up to adding $\varepsilon e_{0}$ we can assume that projections of $v, w$ on $\mathbb{R} e_{0}$ are not trivial. Consider the control:

$$
u=a_{1} e_{1}+\cdots+a_{n-1} e_{n-1}+\lambda v+\mu w
$$

and try to solve equations: $E_{i}(u)=\delta_{i}^{1} T, i=1 \ldots n$.

First from the Implicit Function Theorem we get, solving the $n-1$ first coordinates (i.e. the controllable part of the system):

Now the last coordinate is:

$$
a_{i}=f_{i}(\lambda, \mu)=\mathrm{o}(\lambda, \mu), i=1 \ldots n-1 .
$$

$$
E_{n}(u)=Q(u)+\mathrm{o}\left(\|u\|_{\mathrm{L}^{2}}^{2}\right)=\lambda^{2} Q(v)+\mu^{2} Q(w)+\lambda \mu \check{Q}(v, w)+\mathrm{o}\left(\lambda^{2}, \mu^{2}\right) .
$$

Using the Mean Value theorem we obtain:

- $\exists \lambda_{1}<0, \mu_{1}>0 / E_{n}\left(a_{1} e_{1}+\cdots+a_{n-1} e_{n-1}+\lambda_{1} v+\mu_{1} w\right)=0$;

- $\exists \lambda_{2}>0, \mu_{2}>0 / E_{n}\left(a_{1} e_{1}+\cdots+a_{n-1} e_{n-1}+\lambda_{2} v+\mu_{2} w\right)=0$.

There exists $i=1$ or2 such that $d C_{T}(0) \cdot\left(\lambda_{i} v+\mu_{i} w\right) \neq 0$, and therefore there exists a trajectory steering 0 to $\gamma(T)$ in time $T$ with a cost strictly lower than $C_{T}(0)$.

\subsection{Optimality of abnormal trajectories for sub-Riemannian systems of rank 2}

With the notations of Section 2.2 we have the following result:

Theorem 3.10. Under assumptions of Theorem 2.21, the abnormal reference trajectory $\gamma$ is $\mathrm{C}^{0}$-optimal for the sub-Riemannian system (14) if and only if it is $\mathrm{C}^{0}$-time-minimal for its associated affine system (16). Moreover $\gamma$ is abnormal for this affine system; actually $\gamma$ is $\mathrm{C}^{0}$-optimal if $T<t_{c c}$ and is not $\mathrm{L}^{\infty}$-optimal if $T>t_{c c}$.

In particular conjugate times are the same along $\gamma$ for the sub-Riemannian system (14) and its associated affine system (16). Therefore the whole formalism that was introduced for affine systems (the differential operators $D_{1}, D_{2}$ ) is still valid in sub-Riemannian geometry. Hence the conjugate time of the sub-Riemannian problem can be computed using an algorithm. This result makes a link between works of $[9]$ and $[4,5]$.

Example 3.11. The Martinet case (see Sect. 2.2.2) is in dimension 3, hence $t_{c c}=+\infty$ (see Rem. 3.3). The abnormal trajectory is optimal on $\mathbb{R}^{+}$.

Remark 3.12. As proved in [3] the $\mathrm{C}^{0}$-optimality is in sub-Riemannian geometry equivalent to the optimality in the $\mathrm{L}^{2}$ topology on controls.

Remark 3.13. If $T$ is small enough (depending on the choice of the Riemannian structure, and lower than $t_{c c}$ ), then $\gamma$ is moreover globally optimal among all sub-Riemannian trajectories steering 0 to $\gamma(T)$ (see for instance [4]).

Remark 3.14. It should be noted that the loss of optimality holds in $L^{\infty}$. Hence using the definitions introduced in Section 2.2.2, the $\mathrm{L}^{2}$-sector plays no role in the optimality of the reference abnormal trajectory. The loss of optimality holds in the $\mathrm{L}^{\infty}$-sector. 
Proof of Theorem 3.10. The proof goes in the same spirit as proof of Lemma 2.20. If $\gamma$ is not $\mathrm{C}^{0}$-time-minimal for the sub-Riemannian system (14) at time $T$, then from [4] $\gamma(T)$ is the final point at time $t<T$ of a trajectory associated to a control $\mathrm{L}^{\infty}$-close to the abnormal reference control $v=1, u=0$ :

$$
\forall \varepsilon>0 \quad \exists u, v /|u| \leq \varepsilon,|1-v| \leq \varepsilon, v^{2}+u^{2} \leq 1, \quad \exists t_{f}<T / \gamma(T)=x_{u, v}\left(t_{f}\right) .
$$

In particular: $v<1$. Let us make the following reparametrizing: $\frac{\mathrm{d} s}{\mathrm{~d} t}=v, w(s)=\frac{u(t)}{v(t)}, y(s)=x(t)$. It is valid since $v$ does not vanish on $[0, T]$. We get:

$$
\frac{\mathrm{d} y}{\mathrm{~d} s}=X(y)+w Y(y), y(0)=0
$$

and moreover $w$ satisfies the constraint:

$$
|w| \leq \frac{\varepsilon}{1-\varepsilon} \leq \eta
$$

if $\varepsilon$ is small enough. Hence $y$ is a solution of the affine system (16) with constraint, such that

$$
y\left(s_{f}\right)=x\left(t_{f}\right)=\gamma(T)
$$

where $s_{f}=\int_{0}^{t_{f}} v \mathrm{~d} t<t_{f}<T$ (since $v<1$ ), which proves that $\gamma$ is not $\mathrm{C}^{0}$-time-minimal for the affine system (16).

Conversely, if $\gamma$ is not time-minimal for the affine system (16), there exists a control $w$ with $|w| \leq \eta$ such that the trajectory $y$ associated to $w$ satisfies:

- $\frac{\mathrm{d} y}{\mathrm{~d} s}=X+w Y, y(0)=0$;

- $\exists s_{f}<T / y\left(s_{f}\right)=\gamma(T)$.

Moreover from Remark 3.7 we have: $T-s_{f}=\mathrm{O}\left(a_{n}\right)$. Let $\varepsilon>0$ small (to be chosen), and set $v=1-\varepsilon, u=$ $(1-\varepsilon) w$. We shall prove that $\varepsilon$ can be chosen so that the control $(v, u)$ steers the sub-Riemannian system (14) from 0 to $\gamma(T)$ in time $t_{f}<T$, and satisfies the constraint $v^{2}+u^{2} \leq 1$. Let us make the reparametrizing: $\frac{\mathrm{d} s}{\mathrm{~d} t}=v=1-\varepsilon, x(t)=y(s)$. We obtain:

$$
\frac{\mathrm{d} x}{\mathrm{~d} t}=v X(x)+u Y(x), x(0)=0
$$

and $x\left(t_{f}\right)=y\left(s_{f}\right)=\gamma(T)$, where $s_{f}=(1-\varepsilon) t_{f}$. We have to choose $\varepsilon$ so that the following two conditions are fulfilled: $t_{f}<T$ and $v^{2}+u^{2} \leq 1$ (for then $\gamma$ is not optimal for the sub-Riemannian system 14). From our construction:

Hence we have to impose:

$$
t_{f}=\frac{s_{f}}{1-\varepsilon} \quad \text { and } \quad v^{2}+u^{2} \leq(1-\varepsilon)^{2}\left(1+\eta^{2}\right)
$$

$$
\frac{s_{f}}{1-\varepsilon}<T \quad \text { and } \quad(1-\varepsilon)^{2}\left(1+\eta^{2}\right) \leq 1
$$

i.e.

$$
\varepsilon<1-\frac{s_{f}}{T} \quad \text { and } \quad \varepsilon \geq 1-\frac{1}{\sqrt{1+\eta^{2}}} .
$$

It is possible to choose such an $\varepsilon>0$ provided:

$$
1-\frac{1}{\sqrt{1+\eta^{2}}}<1-\frac{s_{f}}{T} .
$$

From Remark 3.7, we know that: $s_{f}=T-f(\eta)$ if $a_{n}=\mathrm{O}(\eta)$, where $f$ is positive near 0 and $f(\eta)=\mathrm{O}(\eta)$. Hence: $1-\frac{s_{f}}{T}=f(\eta)$. On the other part: $1-\frac{1}{\sqrt{1+\eta^{2}}}=\frac{1}{2} \eta^{2}+o\left(\eta^{2}\right)$. Therefore if $\eta$ is small enough then 
condition (24) is fulfilled. Note that at this point the fact that the optimality of $\gamma$ for the affine system does not depend on the constraint was crucial.

I would like to thank my teacher B. Bonnard for many relevant ideas and advices.

\section{REFERENCES}

[1] A. Agrachev, Compactness for sub-Riemannian length minimizers and subanalyticity. Rend. Sem. Mat. Torino 56 (1998).

[2] A. Agrachev, Quadratic mappings in geometric control theory. J. Soviet Math. 51 (1990) 2667-2734.

[3] A. Agrachev, Any smooth simple $\mathrm{H}^{1}$-local length minimizer in the Carnot-Caratheodory space is a $\mathrm{C}^{0}$-local length minimizer, Preprint. Labo. de Topologie, Dijon (1996).

[4] A. Agrachev and A.V. Sarychev, Strong minimality of abnormal geodesics for 2-distributions. J. Dynam. Control Systems 1 (1995) 139-176.

[5] A. Agrachev and A.V. Sarychev, Abnormal sub-Riemannian geodesics: Morse index and rigidity. Ann. Inst. H. Poincaré 13 (1996) 635-690.

[6] A. Agrachev and A.V. Sarychev, On abnormal extremals for Lagrange variational problems. J. Math. Systems Estim. Control 8 (1998) 87-118.

[7] G.A. Bliss, Lectures on the calculus of variations. U. of Chicago Press (1946).

[8] B. Bonnard and M. Chyba, The role of singular trajectories in control theory. Springer Verlag, Math. Monograph (to be published).

[9] B. Bonnard and I. Kupka, Théorie des singularités de l'application entrée/sortie et optimalité des trajectoires singulières dans le problème du temps minimal. Forum Math. 5 (1993) 111-159.

[10] B. Bonnard and I. Kupka, Generic properties of singular trajectories. Ann. Inst. H. Poincaré Anal. Non Linéaire 14 (1997) 167-186.

[11] B. Bonnard and E. Trélat, On the role of abnormal minimizers in SR-geometry, Preprint. Labo. Topologie Dijon. Ann. Fac. Sci. Toulouse (to be published).

[12] B. Bonnard and E. Trélat, Stratification du secteur anormal dans la sphère de Martinet de petit rayon, edited by A. Isidori, F. Lamnabhi Lagarrigue and W. Respondek. Springer, Lecture Notes in Control and Inform. Sci. 259, Nonlinear Control in the Year 2000, Vol. 2. Springer (2000).

[13] H. Brezis, Analyse fonctionnelle. Masson (1993).

[14] R.L. Bryant and L. Hsu, Rigidity of integral curves of rank 2 distributions. Invent. Math. 114 (1993) 435-461.

[15] M.R. Hestenes, Applications of the theory of quadratic forms in Hilbert space to the calculus of variations. Pacific J. Math. 1 (1951) 525-581.

[16] E.B. Lee and L. Markus, Foundations of optimal control theory. John Wiley, New York (1967).

[17] C. Lesiak and A.J. Krener, The existence and Uniqueness of Volterra Series for Nonlinear Systems. IEEE Trans. Automat. Control AC 23 (1978).

[18] W.S. Liu and H.J. Sussmann, Shortest paths for sub-Riemannian metrics of rank two distributions. Mem. Amer. Math. Soc. 118 (1995).

[19] R. Montgomery, Abnormal minimizers. SIAM J. Control Optim. 32 (1997) 1605-1620.

[20] M.A. Naimark, Linear differential operators. Frederick U. Pub. Co (1967).

[21] L. Pontryagin et al., Théorie mathématique des processus optimaux. Eds Mir, Moscou (1974).

[22] A.V. Sarychev, The index of the second variation of a control system. Math. USSR Sbornik 41 (1982).

[23] E. Trélat, Some properties of the value function and its level sets for affine control systems with quadratic cost. J. Dynam. Control Systems 6 (2000) 511-541.

[24] E. Trélat, Étude asymptotique et transcendance de la fonction valeur en contrôle optimal ; catégorie log-exp dans le cas sous-Riemannien de Martinet, Ph.D. Thesis. Université de Bourgogne, Dijon, France (2000).

[25] Zhong Ge, Horizontal path space and Carnot-Caratheodory metric. Pacific J. Math. 161 (1993) 255-286. 